\title{
Individual versus group strategy-proofness: When do they coincide?
}

\author{
Salvador Barberà ${ }^{\mathrm{a}, \mathrm{b}, \mathrm{c}, *}$, Dolors Berga ${ }^{\mathrm{d}}$, Bernardo Moreno ${ }^{\mathrm{e}}$ \\ ${ }^{a}$ Universitat Autònoma de Barcelona, Edifici B, 08193 Bellaterra, Spain \\ ${ }^{\mathrm{b}}$ MOVE, Universitat Autònoma de Barcelona, Spain \\ ${ }^{\mathrm{c}}$ Barcelona GSE, Spain \\ d Departament d'Economia, Universitat de Girona, Spain \\ e Departamento de Teoría e Historia Económica, Universidad de Málaga, Spain
}

Received 9 July 2009; final version received 16 December 2009; accepted 16 February 2010

\begin{abstract}
A social choice function is group strategy-proof on a domain if no group of agents can manipulate its final outcome to their own benefit by declaring false preferences on that domain. There are a number of economically significant domains where interesting rules satisfying individual strategy-proofness can be defined, and for some of them, all these rules turn out to also satisfy the stronger requirement of group strategy-proofness. We provide conditions on domains guaranteeing that for all rules defined on them, individual and group strategy-proofness become equivalent. We also provide a partial answer regarding the necessity of our conditions.
\end{abstract}

(C) 2010 Published by Elsevier Inc.

\footnotetext{
We are grateful to three anonymous referees and many colleagues for their useful comments in different congresses and workshops where the paper was presented. Salvador Barberà gratefully acknowledges support from the Spanish Ministry of Science and Innovation through grant "Consolidated Group-C" ECO2008-04756, and by the Generalitat de Catalunya, Departament d'Universitats, Recerca i Societat de la Informació through the Distinció per a la Promoció de la Recerca Universitària, grant SGR2009-0419 and the Barcelona GSE Research Network. Dolors Berga acknowledges the support of the Spanish Ministry of Science and Innovation through grant SEJ2007-60671 and of Generalitat de Catalunya, through grant SGR2009-0189. She also acknowledges the Research Recognition Programme of the Barcelona GSE. Bernardo Moreno acknowledges financial support from Junta de Andalucía through Proyecto de Excelencia 522 and the ECO2008-03674 from the Spanish Ministry of Science and Innovation.

* Corresponding author at: Universitat Autònoma de Barcelona, Edifici B, 08193 Bellaterra, Spain. Fax: +34 935813767 .

E-mail addresses: salvador.barbera@uab.cat (S. Barberà), dolors.berga@udg.edu (D. Berga), bernardo@uma.es
} (B. Moreno). 
JEL classification: D71

Keywords: Strategy-proofness; Group strategy-proofness; Sequential inclusion; Single-peaked preferences; Separable preferences

\section{Introduction}

Strategy-proofness is a demanding condition that most mechanisms will fail to satisfy, unless they are defined on properly restricted environments. Group strategy-proofness is an even more stringent requirement, but also a much more attractive one. Indeed, what use would it be to guarantee that no single individual could cheat, if a handful of them could jointly manipulate the system? Yet, the literature on mechanism design has concentrated mostly in analyzing the weakest of these two conditions: after all, it is hard enough to meet, and often impossible except for trivial procedures.

We are now well beyond the disquieting negative message of the Gibbard-Satterthwaite theorem (Gibbard [6] and Satterthwaite [21]). After years of research, we know of a considerable number of instances where non-trivial mechanisms can be found to be strategy-proof when defined on some domains of interest. They include the case of voting when preferences are single-peaked or separable, or when the outcomes are lotteries; they also include families of cost sharing, matching and allocation procedures, again for the case where the preferences of agents are conveniently restricted. When looking at the rich literature on strategy-proof rules over restricted domains, one discovers, somewhat surprisingly, that some of the non-trivial strategyproof mechanisms that arise in these environments are, indeed, also group strategy-proof! It is as if, after a hard search for a solution to the challenge of strategy-proofness, the additional blessing of group strategy-proofness would arise automatically, as an extra gift. Of course, this does not happen in all cases, but it occurs often enough as to warrant the following question. When is it that, once a strategy-proof social choice function is defined, it is necessarily also group strategyproof? More specifically, what are the domains of preferences under which these two conditions become equivalent and collapse into one and the same requirement?

We provide a rather complete solution to that question in voting models. We exhibit conditions on families of preference profiles such that any strategy-proof social choice function whose domain is one of these families will also be necessarily group strategy-proof.

Before proceeding with an outline of our paper let us briefly comment on the literature about group strategy-proofness. The importance of considering deviations by coalitions, and not only by individuals was already remarked in the early literature on strategy-proofness and implementation. See, for example, the early works of Pattanaik [17], the seminal contributions of Green and Laffont [7] and of Dasgupta, Hammond, and Maskin [5], especially Section 4.5. While these papers refer to the characteristics of functions which may be immune to manipulation by coalitions, others exhibit by means of examples the vulnerability of strategy-proof rules to deviations by coalitions, even by very small ones (Pattanaik [17], Barberà [1], Barberà, Sonnenschein, and Zhou [3], and Serizawa [24]).

Although the analysis of specific rules and their different incentive properties in specific contexts is a question of interest, our approach is a different one. Indeed, what we do is to investigate conditions on domains of preferences under which individual and group strategy-proofness become equivalent. This new approach is a natural consequence of the many remarks by different authors on the coincidence between the two conditions in different settings (Barberà and Jack- 
son [2], Klaus [9], Moulin [15], and Pápai [16]; see also Peleg [18] and Peleg and Sudholter [19] for the connections with the related concept of coalition-proofness). A systematic analysis of this question has only one antecedent we know of, a recent paper by Le Breton and Zaporozhets [12], whose results we generalize and extend (for a detailed comparison see Section 3).

Again, notice that our approach is different though complementary to the study of particular rules. We want to argue that getting answers to the type of questions that we raise is important on several grounds. One is intellectual curiosity: once we remark that two conditions become sometimes equivalent, it is attractive to find out when and why. Another reason to ask questions of equivalence is instrumental. Suppose you want to characterize all strategy-proof rules in a domain. This is never an easy task. But the difficulty will be much less in those cases where equivalence holds, because then you can use, from the onset, the formally much stronger requirement of group strategy-proofness.

Our emphasis in this paper is on domains where the equivalence between individual and group strategy-proofness holds for any rule, without any further qualification. But one can also investigate equivalence within classes of rules satisfying additional conditions. For example, consider social choice functions defined on classical exchange economies, with the natural domain restrictions implied by the fact that agents' preferences are selfish, strictly convex and continuous. In this scenario, Barberà and Jackson [2] established the equivalence between individual and group strategy-proofness for those rules satisfying the added condition of non-bossiness. Therefore, our present approach and results can also be seen as a first step in a new line of research that may address similar questions in environments different than the voting situations studied here.

We provide essentially two conditions for domains to imply the equivalence between our two incentive compatibility requirements. The first one, called sequential inclusion, is satisfied by a variety of domains, including classical ones as those of single-peaked or single-dipped preferences. Interestingly this condition is always satisfied in the case of three alternatives.

Our second condition holds for larger domains where not all individual profiles satisfy sequential inclusion, but where an indirect version of this condition holds. Again, that weaker condition is proven to be sufficient for the equivalence between group and individual strategy-proofness. Notice for the sake of completeness that it is satisfied by the universal domain, where equivalence is obvious. We know from the Gibbard-Satterthwaite theorem that in that case the only strategy-proof rules are the dictatorial ones, which are also obviously group strategy-proof. We also discuss other domains where our condition holds and that admit non-dictatorial classes of strategy-proof rules.

Notice that indirect sequential inclusion is weaker than the direct version of the condition. Therefore, the former is a more general sufficient condition for equivalence, and the one to be emphasized if we are interested in the weakest possible requirement.

Yet, direct sequential inclusion is an interesting property per se. First of all, it is the building block leading to the indirect version. Also, it is a single profile condition, and this has several implications. One is that it is in principle easier to check for. The other is that if the condition holds on a domain, it also does in all of its subdomains. We find that results that allow us to make definite statements regarding all rules defined on a set of preferences, and that translate into statements that apply even if the domain happens to only be a subset of them, are of special interest. In particular, notice that as we shrink a given domain to a smaller one, containing only part of the original profiles, new rules may start satisfying the requirement of strategy-proofness. Sequential inclusion guarantees that these emerging rules will also be group strategy-proof.

The last result in the paper (see Section 5) shows that sequential inclusion is essentially a necessary condition for a domain to guarantee equivalence on all of its subdomains. Under mild 
conditions, we prove that if sequential inclusion is violated for some preference profile in a domain, then it is possible to define a strategy-proof rule on a subdomain of it violating group strategy-proofness.

The paper is organized as follows. Section 2 contains the model, the definition of our basic condition of sequential inclusion, and the statement of our first main result; that is, in domains where this condition is satisfied, individual and group strategy-proofness become equivalent. Section 3 introduces the weaker condition of indirect sequential inclusion, and proves it to still be sufficient to guarantee the equivalence of our two incentive compatibility requirements: this is our second basic result. Section 4 provides examples of domains that satisfy sequential inclusion or the indirect version. Section 5 provides the announced result on the necessity of sequential inclusion to achieve equivalence for a domain and all of its subdomains. Section 6 concludes with some directions for further work. Finally, Appendix A collects all the main proofs.

\section{The model and the basic result}

Let $A$ be the set of alternatives and $N=\{1,2, \ldots, n\}$ be the set of agents (with $n \geqslant 2$ ). Let capital letters $S, T \subset N$ denote subsets of agents while lower case letters $s, t$ denote their cardinality.

Let $\mathcal{R}$ be the set of complete, reflexive, and transitive orderings on $A$ and $\mathcal{R}_{i} \subset \mathcal{R}$ be the set of admissible preferences for agent $i \in N$. A preference profile, denoted by $R_{N}=\left(R_{1}, \ldots, R_{n}\right)$, is an element of $\times_{i \in N} \mathcal{R}_{i}$. Preferences will be denoted in two possible ways. When they are part of a given preference profile, we'll use the notation $R_{i} \in \mathcal{R}_{i}$ to denote that these are the preferences of agent $i$ in the profile. In other cases, we want to have a name for a given preference, and our notation must be independent of who holds the preference in question at what profile. Then we will use superscripts, and let $R^{l}$ stand for a specific relation. We may later on attribute that preference to some agents, and then we will write that $R^{l}=R_{i}$ or equivalently, $R_{i}^{l}{ }^{1}$ As usual, we denote by $P_{i}$ and $I_{i}$ the strict and the indifference part of $R_{i}$, respectively. Let $C, S \subset N$ be two coalitions such that $C \subset S$. We will write the subprofile $R_{S}=\left(R_{C}, R_{S \backslash C}\right) \in \times_{i \in S} \mathcal{R}_{i}$ when we want to stress the role of coalition $C$ in $S$. Then the subprofiles $R_{C} \in \times_{i \in C} \mathcal{R}_{i}$ and $R_{S \backslash C} \in \times_{i \in S \backslash C} \mathcal{R}_{i}$ denote the preferences of agents in $C$ and in $S \backslash C$, respectively. In the case, where we denote full preference profile (that is, when $S=N$ ), we simplify notation by using $\left(R_{C}, R_{N \backslash C}\right)$ as $\left(R_{C}, R_{-C}\right)$.

The following concept is crucial in all our analysis. For any $x \in A$ and $R_{i} \in \mathcal{R}_{i}$, define the lower contour set of $R_{i}$ at $x$ as $L\left(R_{i}, x\right)=\left\{y \in A: x R_{i} y\right\}$. Similarly, the strict lower contour set at $x$ is $\bar{L}\left(R_{i}, x\right)=\left\{y \in A: x P_{i} y\right\}$.

A social choice function (or a rule) is a function $f: \times_{i \in N} \mathcal{R}_{i} \rightarrow A$. Let $A_{f}$ denote the range of the social choice function $f$.

We will focus on rules that are nonmanipulable, either by a single agent or by a coalition of agents. We first define what we mean by a manipulation and then we introduce the well-known concepts of strategy-proofness and group strategy-proofness.

Definition 1. A social choice function $f$ is group manipulable on $\times_{i \in N} \mathcal{R}_{i}$ at $R_{N} \in \times_{i \in N} \mathcal{R}_{i}$ if there exists a coalition $C \subset N$ and $R_{C}^{\prime} \in \times_{i \in C} \mathcal{R}_{i}\left(R_{i}^{\prime} \neq R_{i}\right.$ for any $\left.i \in C\right)$ such that $f\left(R_{C}^{\prime}, R_{-C}\right) P_{i} f\left(R_{N}\right)$ for all $i \in C$. We say that $f$ is individually manipulable if there exists a possible manipulation where coalition $C$ is a singleton.

1 The latter notation will be used extensively in Appendix A, proof of Theorem 4. 
Definition 2. A social choice function $f$ is group strategy-proof on $\times_{i \in N} \mathcal{R}_{i}$ if $f$ is not group manipulable for any $R_{N} \in \times_{i \in N} \mathcal{R}_{i}$. Similarly, $f$ is strategy-proof if it is not individually manipulable.

The reader will notice that our definition requires that all agents in a coalition that manipulates should obtain a strictly positive benefit from doing so. We consider this requirement compelling, since it leaves no doubt regarding the incentives for each member of the coalition to participate in a collective deviation from truthful revelation.

Notice that the domains of our social choice functions will always have the form of a Cartesian product. This is necessary to give meaning to our definitions of individual and group strategyproofness. Also notice that, although the notion of a domain is attached to that of a given function, we shall also refer to any Cartesian family of preference profiles as a domain, and to its Cartesian subsets as its subdomains. This is consistent with tradition, although it would be more precise to call them potential domains, as we shall in fact consider sets of functions that could be defined on them.

We shall not dwell on the importance of achieving either of these two incentive compatibility conditions. Our focus will be on these specific cases where it is possible to at least define satisfactory strategy-proof rules. What is needed then to hope for the stronger and much more reassuring property of group strategy-proofness to also hold? This is the main question we address.

Specifically, in this section we define our first condition on preference profiles, called sequential inclusion, and we establish the equivalence between individual and group strategy-proofness for social choice functions defined on domains satisfying that condition. Before that, let us introduce some notation that will be important and will often appear along the paper.

Let $R_{N} \in \times_{i \in N} \mathcal{R}_{i}$, and $y, z$ be a pair of alternatives. Denote by $S\left(R_{N} ; y, z\right) \equiv\left\{i \in N: y P_{i} z\right\}$, that is, the set of agents who strictly prefer $y$ to $z$ according to their individual preferences in $R_{N}$.

Definition 3. Given a preference profile $R_{N} \in \times_{i \in N} \mathcal{R}_{i}$ and a pair of alternatives $y, z \in A$, we define a binary relation $\succsim\left(R_{N} ; y, z\right)$ on $S\left(R_{N} ; y, z\right)$ as follows ${ }^{2}$ :

$$
i \succsim\left(R_{N} ; y, z\right) j \quad \text { if } L\left(R_{i}, z\right) \subset \bar{L}\left(R_{j}, y\right) .
$$

Note that the binary relation $\succsim$ must be reflexive but not necessarily complete. As usual, we can define the strict and the indifference binary relations associated to $\succsim$. Formally, $i \sim j$ if $L\left(R_{i}, z\right) \subset \bar{L}\left(R_{j}, y\right)$ and $L\left(R_{j}, z\right) \subset \bar{L}\left(R_{i}, y\right)$. We say that $i \succ j$ if $L\left(R_{i}, z\right) \subset \bar{L}\left(R_{j}, y\right)$ and $\neg\left[L\left(R_{j}, z\right) \subset \bar{L}\left(R_{i}, y\right)\right]$.

We can now define our main condition.

Definition 4. A preference profile $R_{N} \in \times_{i \in N} \mathcal{R}_{i}$ satisfies sequential inclusion for $y, z \in A$ if the binary relation $\succsim\left(R_{N} ; y, z\right)$ on $S\left(R_{N} ; y, z\right)$ is complete and acyclic.

Definition 5. A preference profile $R_{N} \in \times_{i \in N} \mathcal{R}_{i}$ satisfies sequential inclusion if for any pair $y, z \in A$ the binary relation $\succsim\left(R_{N} ; y, z\right)$ on $S\left(R_{N} ; y, z\right)$ is complete and acyclic. A domain $\times_{i \in N} \mathcal{R}_{i}$ satisfies sequential inclusion if any preference profile in this domain satisfies it.

\footnotetext{
2 In what follows, and when this does not induce to error, we may omit the arguments $R_{N}, y$ and $z$ and just write $\succsim$. Note also that we use the inclusion symbols " $C$ " and " $\supset$ " in a weak sense, that is, they encompass equality of sets.
} 
Table I

Preferences in Example 1.

\begin{tabular}{lll}
\hline$R$ & $R^{\prime}$ & $\widetilde{R}$ \\
\hline $\mathrm{x}$ & $\mathrm{x}$ & $\mathrm{w}$ \\
$\mathbf{y}$ & $\mathbf{y}$ & $\mathbf{y}$ \\
$\mathbf{z}$ & $\mathrm{w}$ & $\mathbf{z}$ \\
$\mathrm{W}$ & $\mathbf{z}$ & $\mathrm{x}$ \\
\hline
\end{tabular}

Table II

Preferences in Example 2.

\begin{tabular}{lll}
\hline$R$ & $R^{\prime}$ & $\widehat{R}$ \\
\hline $\mathrm{x}$ & $\mathrm{w}$ & $\mathrm{t}$ \\
$\mathbf{y}$ & $\mathbf{y}$ & $\mathbf{y}$ \\
$\mathrm{w}$ & $\mathrm{t}$ & $\mathrm{x}$ \\
$\mathbf{z}$ & $\mathbf{z}$ & $\mathbf{z}$ \\
$\mathrm{t}$ & $\mathrm{x}$ & $\mathrm{w}$ \\
\hline
\end{tabular}

Since sequential inclusion is a property on preference profiles, it follows that if a domain satisfies sequential inclusion each subdomain inherits the same property. Remarkably, this condition does not require domains to be large in size, contrary to others, like "richness" (see Dasgupta, Hammond, and Maskin [5], Le Breton and Zaporozhets [12]) or our own condition of indirect sequential inclusion (see Section 3).

Remark 1. Notice that there are two different ways to violate sequential inclusion: by lack of completeness and because of cycles. Both aspects of the definition are essential in what follows, but could be factored out for other purposes, as their implications are different.

To illustrate sequential inclusion and the previous remark consider the following examples.

Example 1. Let $N=\{1,2\}, A=\{x, y, z, w\}$, and $\mathcal{R}_{1}, \mathcal{R}_{2} \supset\left\{R, R^{\prime}, \widetilde{R}\right\}$, where $x P y P z P w$, $x P^{\prime} y P^{\prime} w P^{\prime} z$, and $w \widetilde{P} y \widetilde{P} z \widetilde{P} x$ as represented ${ }^{3}$ in Table I.

Let $R_{N}=\left(R, R^{\prime}\right)$ and take $y, z \in A$. By definition, $S\left(R_{N} ; y, z\right)=\{1,2\}$. Observe also that $L(R, z) \subset \bar{L}\left(R^{\prime}, y\right)$ and $L\left(R^{\prime}, z\right) \subset \bar{L}(R, y)$, that is, $1 \sim\left(R_{N} ; y, z\right) 2$. Thus, $\succsim\left(R_{N} ; y, z\right)$ is complete (trivially acyclic since there are only two agents). The same argument works for any pair of alternatives and thus we can conclude that $R_{N}$ satisfies sequential inclusion.

Let $R_{N}=(R, \widetilde{R})$ and take $y, z \in A$. By definition, $S\left(R_{N} ; y, z\right)=\{1,2\}$. Observe that neither $L(R, z) \subset \bar{L}(\widetilde{R}, y)$ nor $L(\widetilde{R}, z) \subset \bar{L}(R, y)$ holds. Then $\succsim\left(R_{N} ; y, z\right)$ is not complete which means that $R_{N}$ violates sequential inclusion.

Example 2. Let $N=\{1,2,3\}, A=\{x, y, z, w, t\}$, and $\mathcal{R}_{1}, \mathcal{R}_{2} \supset\left\{R, R^{\prime}, \widehat{R}\right\}$ such that the binary relations stated in Table II hold.

Let $R_{N}=\left(R, R^{\prime}, \widehat{R}\right), y, z: S\left(R_{N} ; y, z\right)=\{1,2,3\}$. Observe that $L(R, z) \subset \bar{L}\left(R^{\prime}, y\right)$ but $\neg\left[L\left(R^{\prime}, z\right) \subset \bar{L}(R, y)\right]$, then $1 \succ 2$. Similarly, $2 \succ 3$ and $3 \succ 1$. Thus, $\succsim\left(R_{N} ; y, z\right)$ is complete but it has a cycle: $R_{N}$ violates sequential inclusion.

\footnotetext{
3 From now on, we will use in some examples the same table representation for individual preferences.
} 
Below we present a somewhat surprising result for the case of three alternatives: We show that when there are at most three alternatives at stake, any preference profile $R_{N} \in \times_{i \in N} \mathcal{R}_{i}$ satisfies sequential inclusion.

Proposition 1. Let \#A $\leqslant$ 3. Then, any profile of preferences $R_{N} \in \times_{i \in N} \mathcal{R}_{i}$ satisfies sequential inclusion. $^{4}$

The proof is in Appendix A. Notice that Proposition 1 cannot be generalized for more than three alternatives, as shown by Example 1 above: profiles violating sequential inclusion can be already constructed with four alternatives.

We now present our first main result, which we prove in Appendix A.

Theorem 1. Let $\times_{i \in N} \mathcal{R}_{i}$ be a domain satisfying sequential inclusion. Then, any strategy-proof social choice function on $\times_{i \in N} \mathcal{R}_{i}$ is group strategy-proof.

Proposition 1 and Theorem 1 imply the following corollary.

Corollary 1. Let $\# A \leqslant 3$. Any strategy-proof social choice function on $\times_{i \in N} \mathcal{R}_{i}$ is group strategyproof.

\section{Indirect sequential inclusion}

In this section we provide a new condition, weaker than sequential inclusion, that still guarantees the equivalence between individual and group strategy-proofness. It is no longer a condition on individual profiles. Rather, it requires that, given a profile within the domain, some other profile, conveniently related to the first one (see below), does indeed satisfy our previous requirement. That is why we say that profiles that meet our new condition satisfy indirect sequential inclusion. The new definition allows us to incorporate new and interesting domains into our list of those guaranteeing equivalence.

One of them is the universal domain. It is nice to have this clear case of equivalence incorporated into our framework. Moreover, as we mention below, there are also other relevant domains where sequential inclusion holds indirectly, while not in its direct form.

Definition 6. For preferences $R_{i}, R_{i}^{\prime} \in \mathcal{R}_{i}$ and alternative $z \in A, R_{i}^{\prime}$ is a strict monotonic transformation of $R_{i}$ at $z$ if $R_{i}^{\prime}$ is such that for all $x \in A \backslash\{z\}$ such that $z R_{i} x, z P_{i}^{\prime} x$.

Definition 7. Let $R_{N}^{\prime}, R_{N} \in \times_{i \in N} \mathcal{R}_{i}$ be two preference profiles and let $z \in A$. We say that $R_{N}^{\prime}$ is a strict monotonic transformation of $R_{N}$ at alternative $z$ if for any $i \in N$, either $R_{i}^{\prime}=R_{i}$ or else $R_{i}^{\prime}$ is a strict monotonic transformation of $R_{i}$ at $z$.

Definition 8. A domain $\times_{i \in N} \mathcal{R}_{i}$ satisfies indirect sequential inclusion if, for all profiles $R_{N} \in$ $\times_{i \in N} \mathcal{R}_{i}$, either (a) the profile $R_{N}$ satisfies sequential inclusion; or else (b) for each pair $y, z \in A$ there exists $R_{N}^{\prime} \in \times_{i \in N} \mathcal{R}_{i}$ where $R_{N \backslash S\left(R_{N} ; y, z\right)}^{\prime}=R_{N \backslash S\left(R_{N} ; y, z\right)}$, such that

\footnotetext{
4 The same proof works to show the same result if $\# A_{f} \leqslant 3$.
} 
(1) $R_{N}^{\prime}$ is a strict monotonic transformation of $R_{N}$ at $z$,

(2) for any $i \in S\left(R_{N} ; y, z\right), y P_{i}^{\prime} z$, and

(3) $\succsim\left(R_{N}^{\prime} ; y, z\right)$ is complete and acyclic.

Theorem 2. Let $\times_{i \in N} \mathcal{R}_{i}$ be a domain satisfying indirect sequential inclusion. Then, any strategyproof social choice function on $\times_{i \in N} \mathcal{R}_{i}$ is group strategy-proof.

See the proof of Theorem 2 in Appendix A.

Indirect sequential inclusion is indeed the weakest of our two conditions for equivalence between individual and group strategy-proofness. Still, we pursue the study of both direct and indirect sequential inclusion because each one of the two has its own attractive features. The indirect version is indeed weaker, and thus covers more cases. But since it is a condition on domains taken as a whole, it may fail to hold if some preference profiles are removed from a domain that did satisfy it originally. Direct sequential inclusion is posited on each individual preference profile. Therefore, if it is satisfied on a given domain, it also holds for any of its subdomains. Indeed, it is a stronger condition, but as we shall see in Section 4 , it also allows us to make additional statements of interest.

With our results in hand, we can now comment on the relationship between our paper and Le Breton and Zaporozhets [12]. One obvious addition on our side is everything regarding the condition of sequential inclusion. So we restrict the comparison to the result involving indirect sequential inclusion. To do that, we first adapt to their framework where each individual set of admissible preferences coincides; that is, $\mathcal{R}_{i}=\mathcal{R}$ for any $i \in N$, and then we need two of their definitions, and the statement of their result.

Definition 9. (See Definition 6 in Le Breton and Zaporozhets [12].) A domain $\mathcal{R}^{n}$ is rich if for all $R \in \mathcal{R}$ and any $x, y \in A$ such that $y P x$ and there exists $R_{y} \in \mathcal{R}$ where $y P_{y} z$ for any $z \in A \backslash\{y\}$, then, there exists $R^{\prime} \in \mathcal{R}$ where $y P^{\prime} z$ for any $z \in A \backslash\{y\}$, and for all $z \neq x$ such that $x R z$, we have $x P^{\prime} z$.

Definition 10. (See Definition 3 in Le Breton and Zaporozhets [12].) A social choice function is regular if for any alternative in the range, say $x \in A_{f}$, there exists an individual preference $R_{x} \in \mathcal{R}$ such that $x P_{x} z$ for any $z \in A \backslash\{x\}$.

Theorem 3. (See Theorem 1 in Le Breton and Zaporozhets [12].) If a regular social choice function on a rich domain is strategy-proof, then it is also group strategy-proof.

The comparison of this result with that of our Theorem 2 requires some initial and subtle remarks. First, notice that domains satisfying sequential inclusion (direct or indirect) need not be rich, while rich domains need not satisfy either of our conditions. ${ }^{5}$ Hence, if it was not for the regularity condition, both theorems might have looked independent but in fact they are not. Our second remark is that regularity imposes special connections between the domain of definition of social choice functions and their range. Specifically, there are domains of preferences which admit regular preferences with a full range, and other domains that force the ranges of regular functions to be strictly smaller than the set of alternatives.

\footnotetext{
5 Examples available upon request.
} 
As stated in Proposition 2 below, and proved in Appendix A, any rich domain admitting some regular, onto social choice function must satisfy indirect sequential inclusion. Thus, for these domains, our result strictly improves on Le Breton and Zaporozhets [12].

Proposition 2. Let $\mathcal{R}^{n}$ be a rich domain admitting onto and regular social choice functions. Then, $\mathcal{R}^{n}$ satisfies indirect sequential inclusion.

What if the range of a social choice function satisfying the conditions of Theorem 3 is restricted to be a subset $A^{\prime}$ of $A$ ? Then we could prove that an analog of indirect sequential inclusion holds, weaker but still sufficient to guarantee equivalence between individual and group strategy-proofness.

We omit the definitions and results that would complete our argument, in line with Proposition 2, to keep the paper self contained and within the bounds of editorial recommendations. A companion paper will present further results involving the restriction of our basic concepts here to subsets of voters or to subsets of alternatives.

\section{Examples}

In this section we present examples of domains that illustrate our conditions and results. We discuss some examples in detail, because of their special relevance. At the end of the section we provide a longer list of domains that are also covered by our results.

We first define the domain of single-peaked preferences and we show that it satisfies sequential inclusion. We then define the domain of separable preferences and show that it violates both direct and indirect sequential inclusion.

These two examples of domains are chosen because it is well known that many interesting (non-trivial) strategy-proof social choice functions can be defined on either of them. For the case of single-peaked preferences (see Moulin [13]), all these rules are also group strategy-proof, consistently with our Theorem 1, given that sequential inclusion is satisfied. In contrast, for the case of separable preferences (see Barberà, Sonnenschein, and Zhou [3]), many of these strategy-proof social choice functions are group manipulable, a fact that is again consistent with the violation of indirect sequential inclusion.

After that, we present a third domain, which is given by a proper subset of separable preferences, called lexicographically separable. The domain of separable preferences is, in general, large enough to violate (direct) sequential inclusion, but small enough to respect indirect sequential inclusion. Notice that for the subdomain of lexicographically separable preferences, all of the non-trivial and interesting rules that are known to be individually strategy-proof on the domain of separable preferences would still be, and would now, in addition, satisfy group strategy-proofness.

Finally, we include the case of the universal domain. From the Gibbard-Satterthwaite theorem we already know directly that the equivalence must hold on this domain. Since it only admits dictatorship as a strategy-proof rule, and this is also immune to group manipulation. Even then, we prove that our conditions do hold, just for the sake of completeness.

\subsection{Single-peaked preferences}

Single-peakedness arises as a natural restriction on the preferences of agents facing many relevant problems: determining the level of a pure public good without transfers, locating a facility on a line, deciding on a tax level, choosing among candidates, among others. 
Definition 11. A preference profile $R_{N}$ is single-peaked iff there exists a linear order $>$ of the set of alternatives such that

(1) each of the voters' preferences has a unique maximal element $p_{i}(A)$, called the peak of $i$, and

(2) for all $i \in N$, for all $p_{i}(A)$, and for all $y, z \in A$

$$
\left[p_{i}(A) \geqslant y>z \text { or } z>y \geqslant p_{i}(A)\right] \rightarrow y P_{i} z .
$$

Proposition 3. Any single-peaked profile $R_{N}$ satisfies sequential inclusion.

Proof. Take any single-peaked profile $R_{N}$ and any pair of alternatives $y, z \in A$ such that $z>y$ (if $y>z$ we should interchange the use of the words "left" and "right" in what follows). Let us show that $\succsim\left(R_{N} ; y, z\right)$ on $S\left(R_{N} ; y, z\right)$ is complete and acyclic. Remark that for any pair of agents $i, j$ such that $y P_{i} z$ and $y P_{j} z$, then, either $L\left(R_{i}, z\right) \subset L\left(R_{j}, z\right)$ or $L\left(R_{j}, z\right) \subset L\left(R_{i}, z\right)$. To see this, notice that both lower contour sets are unions of two sets (the set containing alternatives to the right side of $z$ and the set of alternatives to the left side of $y$ ). One of the two sets (the one to the right side of $z$ ) is common to all individual preferences. The other sets are specific to each $z$ and they are nested.

Without loss of generality, say that $L\left(R_{1}, z\right) \subset \cdots \subset L\left(R_{S}, z\right)$. Now order agents in the same way as they appear in this order of lower contour sets. Since $y P_{i} z$ for any $i \in S\left(R_{N} ; y, z\right)$, we have that $1 \succsim 2,3, \ldots, s ; 2 \succsim 3, \ldots, s ; \ldots$; and $s-1 \succsim s$. This shows that $\succsim\left(R_{N} ; y, z\right)$ is complete and acyclic (there is no cycle since for any agent $i, i \succsim j$ for $j$ following $i$ given the order stated by the increasing order of lower contour sets at $z$ ), as required by sequential inclusion.

\subsection{Separable preferences}

The domain of separable preferences is important on the literature of strategy-proofness, since it has been proven to admit rich and attractive classes of non-manipulable rules. Border and Jordan [4], Le Breton and Sen [10], Le Breton and Weymark [11] have analyzed a model with separable preferences in continuous multidimensional spaces. For the purpose of our example, we adopt a simpler framework, where the number of alternatives is finite. It is the one proposed in Barberà, Sonnenschein, and Zhou [3] to analyze the problem of selecting subsets from a set of $k$ objects, $K=\left\{x_{1}, x_{2}, \ldots, x_{k}\right\}$.

Individual preferences are linear orders on the set $2^{K}$ (including the empty set) ${ }^{6}$ Given any preference $R$ on $2^{K}$, we can define the set of "good" objects $G(K, R)=\left\{x_{i} \in K:\left\{x_{i}\right\} P \varnothing\right\}$ and the set of "bad" objects $B(K, R)=\left\{x_{j} \in K: \varnothing P\left\{x_{j}\right\}\right\}$.

Definition 12. $R$ is an individual separable preference on $2^{K}$ if and only if for any set $T$ and any object $x_{l} \notin T, T \cup\left\{x_{l}\right\} P T$ if $x_{l} \in G(K, R)$.

In words, adding a new good object to any set makes the union better than the original set and adding a bad object makes it worse.

\footnotetext{
6 Notice that alternatives, to be denoted by $y, z, \ldots$, are subsets of objects.
} 
Table III

All separable preferences with two objects (Remark 2).

\begin{tabular}{llllllll}
\hline$R^{1}$ & $R^{2}$ & $R^{3}$ & $R^{4}$ & $R^{5}$ & $R^{6}$ & $R^{7}$ & $R^{8}$ \\
\hline$\varnothing$ & $\varnothing$ & $x_{1}$ & $x_{1}$ & $x_{2}$ & $x_{2}$ & $\left\{x_{1}, x_{2}\right\}$ & $\left\{x_{1}, x_{2}\right\}$ \\
$x_{1}$ & $x_{2}$ & $\varnothing$ & $\left\{x_{1}, x_{2}\right\}$ & $\varnothing$ & $\left\{x_{1}, x_{2}\right\}$ & $x_{1}$ & $x_{2}$ \\
$x_{2}$ & $x_{1}$ & $\left\{x_{1}, x_{2}\right\}$ & $\varnothing$ & $\left\{x_{1}, x_{2}\right\}$ & $\varnothing$ & $x_{2}$ & $x_{1}$ \\
$\left\{x_{1}, x_{2}\right\}$ & $\left\{x_{1}, x_{2}\right\}$ & $x_{2}$ & $x_{2}$ & $x_{1}$ & $x_{1}$ & $\varnothing$ & $\varnothing$ \\
\hline
\end{tabular}

Remark 2. Given a set $K$ composed of $k$ objects, the family of all separable preference profiles on $2^{K}$ violates indirect sequential inclusion.

We first offer an indirect argument. We exhibit a rule that it is strategy-proof but not group strategy-proof on these domains. In view of Theorem 2 this proves that indirect sequential inclusion fails.

The rule is voting by quota one: each agent declares her best set of objects and any object that is mentioned by some agent is selected. When $K$ consists of two objects, $x_{1}$ and $x_{2}$, the set of all individual separable preferences is detailed in Table III.

Notice that for a profile where $R_{1}=R^{3}, R_{2}=R^{5}$ and for any other agent $R_{i}=R^{1}$ the outcome would be $\left\{x_{1}, x_{2}\right\}$, whereas 1 and 2 could vote for $\varnothing$ and get a preferred outcome. The example easily extends to any set of alternatives: just take profiles as we have just defined and extend individual preferences such that the relative ordering among $x_{1}, x_{2},\left\{x_{1}, x_{2}\right\}$ and the empty set are like in Table III and any other object is bad.

We could also offer a more direct proof that indirect sequential inclusion does not hold in our domains. We develop the argument for the case $k=2$. Refer to Table III: observe that for any pair of alternatives $y, z$ there only exist four individual separable preferences such that $y$ is strictly preferred to $z$. Thus, for any preference profile $R_{N}, S\left(R_{N} ; y, z\right)$ will always consist of, at most, four different admissible preferences.

To show that the set of separable preferences on $2^{K}$ violates indirect sequential inclusion we need to obtain a profile violating both conditions (a) and (b) in Definition 8. Without loss of generality, consider the following example where $y=\varnothing, z=\left\{x_{1}, x_{2}\right\}$, and suppose that $R_{N}$ is such that the preferences of agents 1 to 4 , are distinct and such that $R_{1}=R^{1}, R_{2}=R^{2}$, $R_{3}=R^{3}$, and $R_{4}=R^{5}$. Remark that this profile does not satisfy sequential inclusion. For the given pair of alternatives, $\succsim\left(R_{N} ; y, z\right)$ is not complete since neither $L\left(R_{3}, z\right) \subset \bar{L}\left(R_{4}, y\right)$ nor $L\left(R_{4}, z\right) \subset \bar{L}\left(R_{3}, y\right)$ and thus, $R_{N}$ does not satisfy condition (a). Finally, notice that there is no admissible monotonic transformation of preferences of $R^{3}$ and $R^{5}$ at $z$ where $y$ is strictly preferred to $z$ (other than $R^{3}$ and $R^{5}$ themselves). Therefore, $R_{N}$ cannot satisfy condition (b).

\subsection{Lexicographically separable preferences}

Now, we introduce an additional restriction on preferences to be added to that of separability. To define this restriction, consider a given order of the objects. Without loss of generality, consider the order $\sigma(k)$ where $x_{1}$ is first, $x_{2}$ is second, etc.

Definition 13. We say that a preference $R$ is lexicographically separable if

(1) it is separable,

(2) all sets containing $x_{1}$ are better (respectively, worse) than any set not containing $x_{1}$, if $x_{1} \in$ $G(K, R)$ (respectively, if $x_{1} \in B(K, R)$ ), 
Table IV

All lexicographically separable preferences with two objects (Remark 3).

\begin{tabular}{llll}
\hline$R^{2}$ & $R^{4}$ & $R^{5}$ & $R^{7}$ \\
\hline$\varnothing$ & $x_{1}$ & $x_{2}$ & $\left\{x_{1}, x_{2}\right\}$ \\
$x_{2}$ & $\left\{x_{1}, x_{2}\right\}$ & $\varnothing$ & $x_{1}$ \\
$x_{1}$ & $\varnothing$ & $\left\{x_{1}, x_{2}\right\}$ & $x_{2}$ \\
$\left\{x_{1}, x_{2}\right\}$ & $x_{2}$ & $x_{1}$ & $\varnothing$ \\
\hline
\end{tabular}

(3) out of sets containing $x_{1}$, those containing $x_{2}$ are better (respectively, worse) than those not containing $x_{2}$, if $x_{2} \in G(K, R)$ (respectively, if $x_{2} \in B(K, R)$ ),

(4) out of sets not containing $x_{1}$, those containing $x_{2}$ are better (respectively, worse) than those not containing $x_{2}$, if $x_{2} \in G(K, R)$ (respectively, if $x_{2} \in B(K, R)$ ),

and so on.

Remark 3. When $k=2$, any profile of lexicographically separable preferences satisfies sequential inclusion.

To substantiate Remark 3, the reader may just inspect the set of all admissible preferences satisfying the restriction when $k=2$, which is detailed in Table IV.

Observe that for any pair of alternatives $y, z$ there only exist two individual separable preferences such that $y$ is strictly preferred to $z$. Thus, for any preference profile $R_{N}, S\left(R_{N} ; y, z\right)$ will always consist of, at most, two different admissible individual preferences. Without loss of generality, consider the pair of alternatives $y=\varnothing, z=\left\{x_{1}, x_{2}\right\}$. Note that the preference of any agent $i \in S\left(R_{N} ; y, z\right)$, is $R_{i} \in\left\{R^{2}, R^{5}\right\}$. Then, we have that for any two agents $i, j \in S\left(R_{N} ; y, z\right)$ if either (a) $R_{i}=R_{j}$ then $L\left(R_{i},\left\{x_{1}, x_{2}\right\}\right) \subset \bar{L}\left(R_{j}, \varnothing\right)$ or (b) if $R_{i} \neq R_{j}$, say $R_{i}=R_{i}^{2}$ and $R_{j}=R_{j}^{5}$, $L\left(R_{i}^{2},\left\{x_{1}, x_{2}\right\}\right)=\left\{x_{1}, x_{2}\right\} \subset \bar{L}\left(R_{j}^{5}, \varnothing\right)=\left\{\left\{x_{1}, x_{2}\right\}, x_{1}\right\}$. Thus, sequential inclusion holds.

Remark 4. When $k>2$, the domain of lexicographically separable preferences (that is, the set of all lexicographically separable preferences profiles) satisfies indirect sequential inclusion but violates sequential inclusion.

We provide an argument supporting the assertions in Remark 4 for the case $k=3$ (this can be generalized for any $k>3$ ). Note that the set of all individual lexicographically separable preferences is as in Table $\mathrm{V}$.

Observe that for any pair of alternatives $y, z$ there only exist four individual lexicographically separable preferences such that $y$ is strictly preferred to $z$. Thus, for any preference profile $R_{N}, S\left(R_{N} ; y, z\right)$ will always consist of, at most, four different admissible individual preferences. Without loss of generality, consider the following example where $y=x_{2}, z=\left\{x_{2}, x_{3}\right\}$, and suppose that $R_{N}$ is such that the preferences of agents 1 to 4 , are distinct and such that $R_{1}=R^{1}, R_{2}=R^{2}, R_{3}=R^{3}$, and $R_{4}=R^{4}{ }^{7}$ Remark that any profile containing these four initial agents would violate sequential inclusion: $\succsim\left(R_{N} ; y, z\right)$ is not complete since neither

\footnotetext{
7 A similar and simpler argument would work for cases where not all distinct admissible preferences are present in the initial profile.
} 
Table V

All lexicographically separable preferences with three objects (Remark 4).

\begin{tabular}{llllllll}
\hline$R^{1}$ & $R^{2}$ & $R^{3}$ & $R^{4}$ & $R^{5}$ & $R^{6}$ & $R^{7}$ & $R^{8}$ \\
\hline$\varnothing$ & $x_{1}$ & $x_{2}$ & $\left\{x_{1}, x_{2}\right\}$ & $x_{3}$ & $\left\{x_{1}, x_{3}\right\}$ & $\left\{x_{2}, x_{3}\right\}$ & $\left\{x_{1}, x_{2}, x_{3}\right\}$ \\
$x_{3}$ & $\left\{x_{1}, x_{3}\right\}$ & $\left\{x_{2}, x_{3}\right\}$ & $\left\{x_{1}, x_{2}, x_{3}\right\}$ & $\varnothing$ & $x_{1}$ & $x_{2}$ & $\left\{x_{1}, x_{2}\right\}$ \\
$x_{2}$ & $\left\{x_{1}, x_{2}\right\}$ & $\varnothing$ & $x_{1}$ & $\left\{x_{2}, x_{3}\right\}$ & $\left\{x_{1}, x_{2}, x_{3}\right\}$ & $x_{3}$ & $\left\{x_{1}, x_{3}\right\}$ \\
$\left\{x_{2}, x_{3}\right\}$ & $\left\{x_{1}, x_{2}, x_{3}\right\}$ & $x_{3}$ & $\left\{x_{1}, x_{3}\right\}$ & $x_{2}$ & $\left\{x_{1}, x_{2}\right\}$ & $\varnothing$ & $x_{1}$ \\
$x_{1}$ & $\varnothing$ & $\left\{x_{1}, x_{2}\right\}$ & $x_{2}$ & $\left\{x_{1}, x_{3}\right\}$ & $x_{3}$ & $\left\{x_{1}, x_{2}, x_{3}\right\}$ & $\left\{x_{2}, x_{3}\right\}$ \\
$\left\{x_{1}, x_{3}\right\}$ & $x_{3}$ & $\left\{x_{1}, x_{2}, x_{3}\right\}$ & $\left\{x_{2}, x_{3}\right\}$ & $x_{1}$ & $\varnothing$ & $\left\{x_{1}, x_{2}\right\}$ & $x_{2}$ \\
$\left\{x_{1}, x_{2}\right\}$ & $x_{2}$ & $x_{1}$ & $\varnothing$ & $\left\{x_{1}, x_{2}, x_{3}\right\}$ & $\left\{x_{2}, x_{3}\right\}$ & $\left\{x_{1}, x_{3}\right\}$ & $x_{3}$ \\
$\left\{x_{1}, x_{2}, x_{3}\right\}$ & $\left\{x_{2}, x_{3}\right\}$ & $\left\{x_{1}, x_{3}\right\}$ & $x_{3}$ & $\left\{x_{1}, x_{2}\right\}$ & $x_{2}$ & $x_{1}$ & $\varnothing$ \\
\hline
\end{tabular}

$L\left(R_{1}, z\right) \subset \bar{L}\left(R_{4}, y\right)$ nor $L\left(R_{4}, z\right) \subset \bar{L}\left(R_{1}, y\right)$. To prove that any profile containing these four agents would respect indirect sequential inclusion we first define a monotonic transform. Simply change the preferences of all individuals whose preferences were initially $R^{1}$ to be now equal to $R^{3}$. Let $\widehat{R}_{N}$ be the new profile. Clearly, $\widehat{R}_{N}$ is a strict monotonic transformation of $R_{N}$ at $z$ and for any $i \in S\left(R_{N} ; y, z\right), y \widehat{P}_{i} z$ (in fact, $S\left(\widehat{R}_{N} ; y, z\right)=S\left(R_{N} ; y, z\right)$ ) and in $\widehat{R}_{N}$ all agents have preferences either $R^{2}$, or $R^{3}$, or $R^{4}$. Moreover, $\widehat{R}_{N}$ satisfies sequential inclusion for $y, z$. To see it, observe that $L\left(\widehat{R}_{i}^{2}, z\right) \subset L\left(\widehat{R}_{j}^{4}, z\right) \subset L\left(\widehat{R}_{k}^{3}, z\right)$. Furthermore, $\neg\left[L\left(\widehat{R}_{k}^{3}, z\right) \subset \bar{L}\left(\widehat{R}_{i}^{2}, y\right)\right]$, $\neg\left[L\left(\widehat{R}_{j}^{4}, z\right) \subset \bar{L}\left(\widehat{R}_{i}^{2}, y\right)\right]$, and $\neg\left[L\left(\widehat{R}_{k}^{3}, z\right) \subset \bar{L}\left(\widehat{R}_{j}^{4}, y\right)\right]$. Thus, $i \succ j$ for any agents $i, j$ such that $R_{i}=R^{2}$ and $R_{j}=R^{3}$, or $R_{i}=R^{2}$ and $R_{j}=R^{4}$, or $R_{i}=R^{4}$ and $R_{j}=R^{3}, i \sim j$ for any agents $i, j$ such that $R_{i}=R_{j}$. Thus, $\succsim\left(\widehat{R}_{N} ; y, z\right)$ is complete and acyclic and the domain where all agents have lexicographically separable preferences on subsets of $k$ objects satisfies indirect sequential inclusion.

\subsection{The universal domain}

Consider the universal domain: for any agent $i \in N, \mathcal{R}_{i}=\mathcal{R}$.

We first notice, that this domain violates sequential inclusion when there are more than three alternatives since we have different examples of subdomains where this condition is not satisfied.

Now, we show that the universal domain satisfies indirect sequential inclusion. Let $R_{N}$ be a preference profile and let $y, z \in A$. If $\succsim\left(R_{N} ; y, z\right)$ is complete and acyclic, let $R_{N}^{\prime}=R_{N}$. Otherwise, define $R_{N}^{\prime}$ as follows: for any $j \in N \backslash S\left(R_{N} ; y, z\right), R_{j}^{\prime}=R_{j}$. And for any $i \in S\left(R_{N} ; y, z\right)$, $R_{i}^{\prime}$ and $R_{i}$ coincide in the ranking of most pairs of alternatives, with two exceptions. One is that $y P_{i}^{\prime} t$ for any $t \in A \backslash\{y\}$ (that is, $y$ is pushed up to be the unique best alternative for $R_{i}^{\prime}$ ). The other change from $R_{i}$ to $R_{i}^{\prime}$ is that for any $t \in A$ such that $t I_{i} z$, then $z P_{i}^{\prime} t$ (that is, alternatives indifferent to $z$ in $R_{i}$ are strictly worse than $z$ under $R_{i}^{\prime}$ ). Observe that $R_{N}^{\prime}$ satisfies the following: for any $i \in S\left(R_{N} ; y, z\right), R_{N}^{\prime}$ is a strict monotonic transformation of $R_{N}$ at $z$. Also, for any agent $i \in S\left(R_{N} ; y, z\right)$ the ranking between $y$ and $z$ has not changed going from $R_{i}$ to $R_{i}^{\prime}$. And finally, $\succsim\left(R_{N}^{\prime} ; y, z\right)$ is complete and acyclic. Observe that $L\left(R_{l}^{\prime}, z\right) \subset \bar{L}\left(R_{i}^{\prime}, y\right)=A \backslash\{y\}$ for any $i, l \in S\left(R_{N} ; y, z\right)$. Thus, $i \sim\left(R_{N} ; y, z\right) l$ for any $i, l \in S\left(R_{N} ; y, z\right)$. This shows that the universal domain satisfies indirect sequential inclusion.

In this last part of this examples section, we provide a list of domains where at least one of our conditions for equivalence is satisfied. We provide some references where the interested reader find the exact definition of the domains we refer to. 


\subsection{Some other examples}

Domains of preferences where sequential inclusion holds, and for which Theorem 1 applies, include:

(i) any subset of single-peaked preferences;

(ii) any subset of single-dipped preferences (see Sen and Pattanaik [23]);

(iii) any subset of clockwise and/or counterclockwise preferences on a finite set of alternatives around a circle (see Kim and Roush [8]);

(iv) any subset of (weakly) single-plateaued preferences (even allowing for some indifferences among alternatives that are not best) (see Moulin [14]);

(v) any subset of (weakly) single-throughed preferences;

(vi) any subset of lexicographically separable preferences on sets of two candidates (see Remark 3 in Section 4).

Domains of preferences where indirect sequential inclusion, though not sequential inclusion, holds, and thus result in Theorem 2 applies, are those generated by the following sets of admissible preferences for any agent:

(vii) the universal domain;

(viii) the case where the preferences of some agents are always single-peaked and those of others always single-dipped (see W. Thomson, Where should your daughter go to college? An axiomatic analysis, mimeo, University of Rochester, 2008);

(ix) lexicographically separable preferences on three or more objects (see Remark 4 in Section 4);

(x) the set of single-crossing preferences when the set of preferences is maximal (see Saporiti [20]);

(xi) the set of all quadratic preferences on some subset $A$ in $\mathbb{R}^{m}$ (see Border and Jordan [4]);

(xii) the set of all single-peaked preferences over the circle when we can travel clockwise or counterclockwise (see Schummer and Vohra [22]).

\section{A partial result on necessity}

In this section we provide a result that establishes the partial necessity of sequential inclusion to guarantee that individual and group strategy-proofness become equivalent. We have already remarked that, if sequential inclusion holds in some domain, it also holds in all subdomains, and so does equivalence. What we can prove is that under additional conditions if equivalence holds for a domain and all of its subdomains, then sequential inclusion must be satisfied by all of them. Or, equivalently, that if sequential inclusion fails for some domain, then it is possible to find a subdomain where to define a rule that is individually but not group strategy-proof. Since this rule will be defined on a subdomain, rather than on the full domain we start with, we do not have a full proof of necessity.

The proof is technically challenging. Since we must start from a violation of sequential inclusion and this may happen due to the existence of cycles of any length $k$, our construction must refer to rules whose range is large enough (as shown in the proof, it must contain at least $k+2$ alternatives). Yet the existence of strategy-proof rules with range of a given size is not easy to guarantee unless we impose some conditions on the nature of the domain. In particular, it is 
easy to find reasonable domains for which the only strategy-proof rules (including the dictatorial ones) require a very small range. Because of that, and since our proof is going to be constructive, we must impose some condition on their structure of the domains in order to get our necessity result. We have explored several such requirements and the result we present here depends on the condition that our domains $\times_{i \in N} \mathcal{R}_{i}$ allow for opposite preferences, that is, are such that for any $i$, if $R_{i} \in \mathcal{R}_{i}$, its opposite $\bar{R}_{i}$ also belongs to $\mathcal{R}_{i}\left(R_{i}\right.$ and $\bar{R}_{i}$ are opposite if for any $x, y \in A$, $\left.x R_{i} y \Leftrightarrow y \bar{R}_{i} x\right)$.

Admittedly, this is a restrictive condition. In particular, it does not hold for some of the domains, like single-peakedness, that we have shown to satisfy sequential inclusion. But it is satisfied by many of the domains where indeed indirect sequential inclusion does not hold. For example, it holds for the set of separable preferences, and also for the domain of linear preferences on simplices, where the alternatives are interpreted as lotteries over pure alternatives. We know of other conditions under which a similar result could be proven and we hope that the reader will be able to appreciate the flavor of the result in addition to the precise presentation that follows.

We now state the basic theorem.

Theorem 4. Let $\times_{i \in N} \mathcal{R}_{i}$ be a domain that allows for opposite preferences and such that any strategy-proof social choice function on $\times_{i \in N} \mathcal{D}_{i} \subset \times_{i \in N} \mathcal{R}_{i}$ is also group strategy-proof. Then, $\times_{i \in N} \mathcal{R}_{i}$ satisfies sequential inclusion.

The proof of Theorem 4 is long and quite involved. We sketch it here and we provide all the remaining details in Appendix A.

Sketch of the proof of Theorem 4. Our starting point is given by a domain of preferences containing some profile where sequential inclusion is violated. We first use this profile in order to identify a specific set of alternatives and a specific set of preferences on these alternatives that belong to the domain and satisfy a number of properties (Lemmas 1 and 2). We use these preferences to create a subdomain of the original one, and then exploit their characteristics in order to construct a specific rule that is strategy-proof but not group strategy-proof, within the subdomain. In the case where sequential inclusion is violated by lack of completeness Lemma 3 provides a simple construction. When the violation is due to a cycle our choice of domain is more involved and the rule is more complex. It is essentially a modification of the Borda rule with two extra features: ties are always avoided and the rule does not follow Borda in two crucial profiles. This rule turns out to be individually but not group strategy-proof on our domain and this completes the proof.

\section{Concluding remarks}

In this paper we have identified conditions on domains where all rules satisfying strategyproofness must also be immune to manipulations by groups. We have not imposed any further conditions on the nature and properties of such rules. One could think of further refinements of our results, where the equivalence between individual and group strategy-proofness might hold (in given domains) between classes of rules which satisfy some additional condition. As mentioned in the Introduction, an example in this line is given by social choice functions defined on classical exchange economies, where Barberà and Jackson [2] have established the equivalence between individual and group strategy-proofness for those rules satisfying the added condition of non-bossiness. 
This example also allows us to comment on the type of domain restrictions we may be interested in. Those that we have used as examples arise very naturally in voting contexts. But there are also other domains that allow for non-trivial strategy-proof rules and that appear in connection with economic problems like those of matching, cost allocation, or the assignment of indivisibles. We conjecture that one would also be able to prove interesting equivalence results within these domains, especially if concentrating in subclasses of functions satisfying appropriate additional conditions.

Also notice that the requirement on the binary relation defining our different notions of sequential inclusion can be factored out into two pieces. One requires this relation to be complete; the other demands its acyclicity. Because of that, in the presence of domain restrictions arising from other considerations, we may not need to impose the full form of sequential inclusion in order to get equivalence. For example, in Cartesian domains where preferences satisfy singlecrossing, satisfying completeness will be sufficient to guarantee indirect sequential inclusion since acyclicity does always hold. In particular, we can prove that completeness will hold on maximal sets of single-crossing preferences (as defined by Saporiti [20]) and this is why we have mentioned these sets of preferences as example (x) in Section 4.

In summary, our work has studied the pure case of equivalence without further restrictions. What we suggest is that imposing added structure on domains and/or the functions defined upon them is a natural next step.

Finally, let us mention that our conditions of direct and indirect sequential inclusion can be weakened in two different ways. On the one hand, requiring the non-existence of cycles of sizes smaller than a fixed $k, k \leqslant n$, and on the other hand imposing our properties on subsets of alternatives, not necessarily on the whole set of alternatives. Using such weakened properties, our results extend to intermediate versions of strategy-proofness, defined to exclude manipulations by small group of agents, and also to state the equivalence when applied to some subsets of alternatives. These conditions and their implications are part of a companion paper (see S. Barberà, D. Berga, B. Moreno, On the design of group strategy-proof mechanisms: domains, ranges and special conditions, mimeo, 2010).

\section{Appendix A. Proofs}

Proof of Proposition 1. If $\# A=2$ or $\# A=1$ sequential inclusion trivially holds. Therefore, we concentrate on the case where $A$ consists of three distinct alternatives $x, y$, and $z$ and $R_{N} \in \times_{i \in N} \mathcal{R}_{i}$. Without loss of generality, choose two of these three alternatives, say $y$ and $z$. Define the following partition: $S\left(R_{N} ; y, z\right)=S_{z}\left(R_{N} ; y, z\right) \cup S_{\{x, z\}}\left(R_{N} ; y, z\right)$ where $S_{z}\left(R_{N} ; y, z\right)=$ $\left\{j \in S\left(R_{N} ; y, z\right)\right.$ such that $\left.L\left(R_{j}, z\right)=\{z\}\right\}$ and $S_{\{x, z\}}\left(R_{N} ; y, z\right)=\left\{k \in S\left(R_{N} ; y, z\right)\right.$ such that $\left.L\left(R_{k}, z\right)=\{x, z\}\right\}$, respectively. Since their lower contour set at $z$ coincide, for any $j, l \in$ $S_{z}\left(R_{N} ; y, z\right), j \sim\left(R_{N} ; y, z\right) l$. Similarly, for any $k, h \in S_{\{x, z\}}\left(R_{N} ; y, z\right), k \sim\left(R_{N} ; y, z\right) h$. Moreover, for any $j \in S_{z}\left(R_{N} ; y, z\right),\{z\}=L\left(R_{j}, z\right) \subset \bar{L}\left(R_{k}, y\right)$ for any $k \in S_{\{x, z\}}\left(R_{N} ; y, z\right)$, thus $j \succsim\left(R_{N} ; y, z\right) k$. Thus, $\succsim\left(R_{N} ; y, z\right)$ is complete and acyclic showing that sequential inclusion holds.

We now prove Theorem 1.

Proof of Theorem 1. We will use the following fact several times: for any reflexive, complete and acyclic binary relation $T$ on a finite set $B$, the set $C(B, T)=\{x \in B: x T y$ for any $y \in B\}$ of best elements of $T$ on $B$ is non-empty. 
Let $f$ be a strategy-proof social choice function on $\times_{i \in N} \mathcal{R}_{i}$ satisfying sequential inclusion. By contradiction, suppose that $f$ is manipulable by some coalition $C \subset N$. That is, there exists a coalition $C, R_{N} \in \times_{i \in N} \mathcal{R}_{i}$, and $\widetilde{R}_{C} \in \times_{i \in C} \mathcal{R}_{i}$, such that for any agent $i \in C$, $f\left(\widetilde{R}_{C}, R_{-C}\right) P_{i} f\left(R_{N}\right)$. Let $y=f\left(\widetilde{R}_{C}, R_{-C}\right)$ and $z=f\left(R_{N}\right)$. Note that $C \subset S\left(R_{N} ; y, z\right)$. Now we will go from $R_{N}$ to $\left(\widetilde{R}_{C}, R_{-C}\right)$ by steps, replacing each time the preference of one agent in $C$. The order in $C$ will be determined by sequential inclusion.

By sequential inclusion, $\succsim\left(R_{N} ; y, z\right)$ is complete and acyclic on $S\left(R_{N} ; y, z\right)$, thus, complete and acyclic on $C$. Choose an agent in $C\left(C ; \succsim\left(R_{N} ; y, z\right)\right)$, without loss of generality, say it is agent 1 . Thus, $L\left(R_{1}, z\right) \subset \bar{L}\left(R_{h}, y\right)$ for $h \in C \backslash\{1\}$. Change preferences of agent 1 and let $z_{1}=$ $f\left(\widetilde{R}_{1}, R_{-\{1\}}\right)$. By strategy-proofness, $z_{1} \in L\left(R_{1}, z\right)$ and thus $z_{1} \in \bar{L}\left(R_{h}, y\right)$ for any $h \in C \backslash\{1\}$. Observe that $S\left(\left(\widetilde{R}_{1}, R_{-\{1\}}\right) ; y, z_{1}\right) \supset C \backslash\{1\}$.

By sequential inclusion, $\succsim\left(R_{N} ; y, z_{1}\right)$ is complete and acyclic on $S\left(R_{N} ; y, z_{1}\right)$, thus, complete and acyclic on $C \backslash\{1\}$. Choose an agent in $C\left(C \backslash\{1\} ; \succsim\left(R_{N} ; y, z_{1}\right)\right)$, without loss of generality, say it is agent 2 . Thus, $L\left(R_{2}, z_{1}\right) \subset \bar{L}\left(R_{h}, y\right)$ for $h \in C \backslash\{1,2\}$. Change preferences of agent 2 and let $z_{2}=f\left(\widetilde{R}_{\{1,2\}}, R_{-\{1,2\}}\right)$. By strategy-proofness, $z_{2} \in L\left(R_{2}, z_{1}\right)$ and thus $z_{2} \in \bar{L}\left(R_{h}, y\right)$ for any $h \in C \backslash\{1,2\}$. Observe that $S\left(\left(\widetilde{R}_{\{1,2\}}, R_{-\{1,2\}}\right) ; y, z_{2}\right) \supset C \backslash\{1,2\}$.

Repeat the same argument changing from $R_{i}$ to $\widetilde{R}_{i}$ the preferences of all agents in $C$. Suppose that we have repeated this process $c-2$ times, that is, without loss of generality, we have changed preferences of agent $c-2$.

By sequential inclusion, $\succsim\left(R_{N} ; y, z_{c-2}\right)$ is complete and acyclic on $S\left(R_{N} ; y, z_{c-2}\right)$, thus, complete and acyclic on $C \backslash\{1, \ldots, c-2\}$. Choose an agent in $C(C \backslash\{1, \ldots, c-2\}$; $\left.\left(R_{N} ; y, z_{c-2}\right)\right)$, without loss of generality, say it is agent $c-1$. Thus, $L\left(R_{c-1}, z_{c-2}\right) \subset$ $\bar{L}\left(R_{h}, y\right)$ for $h \in C \backslash\{1, \ldots, c-1\} \equiv c$. Change preferences of agent $c-1$ and let $z_{c-1}=$ $f\left(\widetilde{R}_{\{1, \ldots, c-1\}}, R_{-\{1, \ldots, c-1\}}\right)$. By strategy-proofness, $z_{c-1} \in L\left(R_{c-1}, z_{c-2}\right)$ and thus $z_{c-1} \in$ $\bar{L}\left(R_{c}, y\right)$.

We now prove our more general sufficiency result.

Proof of Theorem 2. We begin the proof by stating and proving an auxiliary lemma and a remark.

Lemma. Let $f$ be a strategy-proof social choice function. For any $R_{N} \in \times_{i \in N} \mathcal{R}_{i}$, any $i \in N$, if $R_{i}^{\prime} \in \mathcal{R}_{i}$ is a strict monotonic transformation of $R_{i}$ at $f\left(R_{N}\right)$ we have that $f\left(R_{N}\right)=f\left(R_{i}^{\prime}, R_{-i}\right)$.

Proof. Let $f$ be any strategy-proof social choice function. Let $R_{N} \in \times_{i \in N} \mathcal{R}_{i}$ and let $R_{i}^{\prime} \in \mathcal{R}_{i}$ be a strict monotonic transformation of $R_{i}$ at $f\left(R_{N}\right)$. Suppose that $f\left(R_{N}\right) \neq f\left(R_{i}^{\prime}, R_{-i}\right)$. By strategy-proofness, $f\left(R_{N}\right) R_{i} f\left(R_{i}^{\prime}, R_{-i}\right)$ and $f\left(R_{i}^{\prime}, R_{-i}\right) R_{i}^{\prime} f\left(R_{N}\right)$ for all $R_{-i} \in \mathcal{R}_{-i}$. Since $R_{i}^{\prime}$ is a strict monotonic transformation of $R_{i}$ at $f\left(R_{N}\right)$, we have that $f\left(R_{N}\right) P_{i}^{\prime} f\left(R_{i}^{\prime}, R_{-i}\right)$, a contradiction. This ends the proof of the Lemma.

Remark. Notice that condition (b) in Definition 8 can be restated as follows: $\left(b^{\prime}\right)$ for each pair $y, z \in A$ and for each subset $T$ in $S\left(R_{N} ; y, z\right)$, there exists $R_{N}^{\prime} \in \times_{i \in N} \mathcal{R}_{i}$ where $R_{N \backslash T}^{\prime}=R_{N \backslash T}$, such that

(1') $R_{N}^{\prime}$ is a strict monotonic transformation of $R_{N}$ at $z$,

(2') for any $i \in T, y P_{i}^{\prime} z$ and

$\left(3^{\prime}\right) \succsim\left(R_{N}^{\prime} ; y, z\right)$ is complete and acyclic on $T$. 
Proof. Clearly condition (b') implies condition (b). Now, consider $R_{N} \in \times_{i \in N} \mathcal{R}_{i}$ and let $y, z$, and $R_{N}^{\prime}$ such that (b) holds. Let $T \subset S\left(R_{N} ; y, z\right)$ and define $\bar{R}_{N}^{\prime}$ such that $\bar{R}_{N \backslash T}^{\prime}=R_{N \backslash T}$ and $\bar{R}_{T}^{\prime}=R_{T}^{\prime}$. Note that $\left(1^{\prime}\right),\left(2^{\prime}\right)$, and $\left(3^{\prime}\right)$ hold for $\bar{R}_{N}^{\prime}$ on $T$ since (1), (2), and (3) hold for $R_{N}^{\prime}$ on the larger set $S\left(R_{N} ; y, z\right)$. This ends the proof of the Remark.

We now complete the proof of Theorem 2.

Let $f$ be a strategy-proof social choice function on $\times_{i \in N} \mathcal{R}_{i}$ and by contradiction suppose that $f$ is manipulable by some coalition $C \subset N$, that is, there exists $\widetilde{R}_{C} \in \times_{i \in C} \mathcal{R}_{i}$, such that for any agent $i \in C, f\left(\widetilde{R}_{C}, R_{-C}\right) P_{i} f\left(R_{N}\right)$. Let us denote $y=f\left(\widetilde{R}_{C}, R_{-C}\right)$ and $z=f\left(R_{N}\right)$. Note that $C \subset S\left(R_{N} ; y, z\right)$. The proof consists of $c$ steps. Mainly, in each one, we change the preference of a single agent $i \in C$ to be $\widetilde{R}_{i}$.

\section{Step 1:}

Case 1.1. If $R_{N}$ satisfies sequential inclusion. We get a contradiction using the same argument as in the proof of Theorem 1.

Case 1.2. If $R_{N}$ does not satisfy sequential inclusion, since the domain satisfies indirect sequential inclusion, by the above Remark applied to $y, z$ and $T=C$, there exists $R_{N}^{\prime}$ such that $R_{N \backslash C}^{\prime}=$ $R_{N \backslash C}$ and conditions $\left(1^{\prime}\right),\left(2^{\prime}\right)$, and $\left(3^{\prime}\right)$ in the above Remark hold. By the above Lemma and ( $\left.1^{\prime}\right)$, we obtain that $f\left(R_{C}^{\prime}, R_{-C}\right)=z$. By condition $\left(2^{\prime}\right), y=f\left(\widetilde{R}_{C}, R_{-C}\right) P_{i}^{\prime} f\left(R_{C}^{\prime}, R_{-C}\right)=z$. Thus, coalition $C$ manipulates $f$ at $\left(R_{C}^{\prime}, R_{-C}\right)$ via $\widetilde{R}_{C}$. By $\left(3^{\prime}\right), \succsim\left(R_{N}^{\prime} ; y, z\right)$ is complete and acyclic on $C$. Note that $S\left(\left(R_{C}^{\prime}, R_{-C}\right) ; y, z\right)=S\left(R_{N} ; y, z\right)$. Choose an agent in $C\left(C, \succsim\left(R_{N}^{\prime} ; y, z\right)\right)$, without loss of generality, say agent 1 . Then, $L\left(R_{1}^{\prime}, z\right) \subset \bar{L}\left(R_{h}^{\prime}, y\right)$ for any $h \in C \backslash\{1\}$. Change preferences of agent 1 and let $z_{2}=f\left(\widetilde{R}_{1}, R_{C \backslash\{1\}}^{\prime}, R_{-C}\right)$. By strategy-proofness, $z_{2} \in L\left(R_{1}^{\prime}, z\right)$ and thus $z_{2} \in \bar{L}\left(R_{h}^{\prime}, y\right)$ for any $h \in C \backslash\{1\}$. Moreover, observe that $S\left(\left(\widetilde{R}_{1}, R_{C \backslash\{1\}}^{\prime}, R_{-C}\right) ; y, z_{2}\right) \supset C \backslash\{1\}$. Go to Step 2.

Step 2: Redefine $R_{N} \equiv\left(\widetilde{R}_{1}, R_{C \backslash\{1\}}^{\prime}, R_{-C}\right)$.

Case 2.1. If $R_{N}$ satisfies sequential inclusion we get a contradiction using the same argument as in the proof of Theorem 1 changing preferences of all agents except 1 going from $R_{N} \equiv\left(\widetilde{R}_{1}, R_{C \backslash\{1\}}^{\prime}, R_{-C}\right)$ to $\left(\widetilde{R}_{C}, R_{-C}\right)$ and obtaining a contradiction that $C$ manipulates $f$ at $\left(R_{C}^{\prime}, R_{-C}\right)$ via $\widetilde{R}_{C}$.

Case 2.2. If $R_{N}$ does not satisfy sequential inclusion, since the domain satisfies indirect sequential inclusion, by the above Remark applied to $y, z_{2}$ and $T=C \backslash\{1\} \subset S\left(R_{N} ; y, z_{2}\right)=$ $S\left(\left(\widetilde{R}_{1}, R_{C \backslash\{1\}}^{\prime}, R_{-C}\right) ; y, z_{2}\right)$, there exists $R_{N}^{\prime}$ such that $R_{N \backslash[C \backslash\{1\}]}^{\prime}=R_{N \backslash[C \backslash\{1\}]}$ (that is, $\left.R_{\{1\} \cup N \backslash C}^{\prime}=\left(\widetilde{R}_{1}, R_{N \backslash C}\right)\right)$ such that conditions $\left(1^{\prime}\right),\left(2^{\prime}\right)$, and $\left(3^{\prime}\right)$ in the above Remark hold. By the above Lemma and $\left(1^{\prime}\right)$, we obtain that $f\left(R_{C \backslash\{1\}}^{\prime}, R_{\{1\} \cup N \backslash C}\right)=z_{2}$. By condition $\left(2^{\prime}\right)$, $y=f\left(\widetilde{R}_{C}, R_{-C}\right) P_{i}^{\prime} f\left(R_{C \backslash\{1\}}^{\prime}, R_{\{1\} \cup N \backslash C}\right)=z_{2}$. Thus, coalition $C \backslash\{1\}$ manipulates $f$ at $\left(R_{C \backslash\{1\}}^{\prime}\right.$, $\left.R_{\{1\} \cup N \backslash C}\right)=\left(\widetilde{R}_{1}, R_{C \backslash\{1\}}^{\prime}, R_{-C}\right)$ via $\widetilde{R}_{C \backslash\{1\}}$. By $\left(3^{\prime}\right), \succsim\left(R_{N}^{\prime} ; y, z_{2}\right)$ is complete and acyclic on $C \backslash\{1\}$. Choose an agent in $C\left(C \backslash\{1\}\right.$, $\left.\left(R_{N}^{\prime} ; y, z_{2}\right)\right)$, without loss of generality, say agent 2 . Then, $L\left(R_{2}^{\prime}, z_{2}\right) \subset \bar{L}\left(R_{h}^{\prime}, y\right)$ for any $h \in C \backslash\{1,2\}$. Change preferences of agent 2 and let $z_{3}=f\left(\widetilde{R}_{\{1,2\}}, R_{C \backslash\{1,2\}}^{\prime}, R_{-C}\right)$. By strategy-proofness, $z_{3} \in L\left(R_{2}^{\prime}, z_{2}\right)$. Thus $z_{3} \in \bar{L}\left(R_{h}^{\prime}, y\right)$ for any $h \in C \backslash\{1,2\}$. Also note that $S\left(\left(\widetilde{R}_{\{1,2\}}, R_{C \backslash\{1,2\}}^{\prime}, R_{-C}\right) ; y, z_{3}\right) \supset C \backslash\{1,2\}$. Go to Step 3 .

Step 3: Redefine $R_{N} \equiv\left(\widetilde{R}_{\{1,2\}}, R_{C \backslash\{1,2\}}^{\prime}, R_{-C}\right)$. 
Case 3.1. If $R_{N}$ satisfies sequential inclusion we get a contradiction using the same argument as in the proof of Theorem 1 applied to $C \backslash\{1\}$ changing preferences of all agents except 2 going from $R_{N} \equiv\left(\widetilde{R}_{\{1,2\}}, R_{C \backslash\{1,2\}}^{\prime}, R_{-C}\right)$ to $\left(\widetilde{R}_{C}, R_{-C}\right)$ and obtaining a contradiction that coalition $C \backslash\{1\}$ manipulates $f$ at $\left(\widetilde{R}_{1}, R_{C \backslash\{1\}}^{\prime}, R_{-C}\right)$ via $\widetilde{R}_{C \backslash\{1\}}$.

Case 3.2. If $R_{N}$ does not satisfy sequential inclusion, since the domain satisfies indirect sequential inclusion, by the above Remark applied to $y, z_{3}$ and $T=C \backslash\{1,2\} \subset S\left(R_{N} ; y, z_{3}\right)=$ $S\left(\left(\widetilde{R}_{\{1,2\}}, R_{C \backslash\{1,2\}}^{\prime}, R_{-C}\right) ; y, z_{3}\right)$, there exists $R_{N}^{\prime}$ such that $R_{N \backslash[C \backslash\{1,2\}]}^{\prime}=R_{N \backslash C \backslash\{1,2\}]}$ (that is, $\left.R_{\{1,2\} \cup N \backslash C}^{\prime}=\left(\widetilde{R}_{\{1,2\}}, R_{N \backslash C}\right)\right)$ such that conditions $\left(1^{\prime}\right),\left(2^{\prime}\right)$, and $\left(3^{\prime}\right)$ in the above Remark hold. By the above Lemma and $\left(1^{\prime}\right)$, we obtain that $f\left(R_{C \backslash\{1,2\}}^{\prime}, R_{\{1,2\} \cup N \backslash C}\right)=z_{3}$. By condition ( $\left.2^{\prime}\right)$, for $i \in C \backslash\{1,2\}, y=f\left(\widetilde{R}_{C}, R_{-C}\right) P_{i}^{\prime} f\left(R_{C \backslash\{1,2\}}^{\prime}, R_{\{1,2\} \cup N \backslash C}\right)=z_{3}$. Thus, coalition $C \backslash\{1,2\}$ manipulates $f$ at $\left(R_{C \backslash\{1,2\}}^{\prime}, R_{\{1,2\} \cup N \backslash C}\right)=\left(\widetilde{R}_{\{1,2\}}, R_{C \backslash\{1,2\}}^{\prime}, R_{-C}\right)$ via $\widetilde{R}_{C \backslash\{1,2\}}$. By $\left(3^{\prime}\right), \succsim\left(R_{N}^{\prime} ; y, z_{3}\right)$ is complete and acyclic on $C \backslash\{1,2\}$. Choose an agent in $C\left(C \backslash\{1,2\}, \succsim\left(R_{N}^{\prime} ; y, z_{3}\right)\right)$, without loss of generality, say agent 3 . Then, $L\left(R_{3}^{\prime}, z_{3}\right) \subset \bar{L}\left(R_{h}^{\prime}, y\right)$ for any $h \in C \backslash\{1,2,3\}$. Change preferences of agent 3 and let $z_{4}=f\left(\widetilde{R}_{\{1,2,3\}}, R_{C \backslash\{1,2,3\}}^{\prime}, R_{-C}\right)$. By strategy-proofness, $z_{4} \in L\left(R_{3}^{\prime}, z_{3}\right)$. Thus $z_{4} \in \bar{L}\left(R_{h}^{\prime}, y\right)$ for any $h \in C \backslash\{1,2,3\}$. Also note that $S\left(\left(\widetilde{R}_{\{1,2,3\}}, R_{C \backslash\{1,2,3\}}^{\prime}, R_{-C}\right) ; y, z_{4}\right) \supset$ $C \backslash\{1,2,3\}$. Go to Step 4 .

We repeat a similar argument till Step $(c-1)$. Note that by the recursive argument when analyzing Step $(c-1)$, from Step $(c-2)$ we know that coalition $C \backslash\{1, \ldots, c-3\}$ manipulates $f$ at $\left(\widetilde{R}_{\{1, \ldots, c-3\}}, R_{C \backslash\{1, \ldots, c-3\}}^{\prime}, R_{-C}\right)$ via $\widetilde{R}_{C \backslash\{1, \ldots, c-3\}}$. Let $z_{c-1}=f\left(\widetilde{R}_{\{1, \ldots, c-2\}}, R_{C \backslash\{1, \ldots, c-2\}}^{\prime}, R_{-C}\right)$. By strategy-proofness, $z_{c-1} \in L\left(R_{c-2}^{\prime}, z_{c-2}\right)$. Thus $z_{c-1} \in \bar{L}\left(R_{h}^{\prime}, y\right)$ for any agent in $\{c-1, c\}$. Moreover, $S\left(\left(\widetilde{R}_{\{1, \ldots, c-2\}}, R_{C \backslash\{1, \ldots, c-2\}}^{\prime}, R_{-C}\right) ; y, z_{c-1}\right) \supset\{c-1,-c\}$.

Step (c-1): Redefine $R_{N} \equiv\left(\widetilde{R}_{\{1, \ldots, c-2\}}, R_{C \backslash\{1, \ldots, c-2\}}^{\prime}, R_{-C}\right)$.

Case (c-1).1. If $R_{N}$ satisfies sequential inclusion we get a contradiction using the same argument as in the proof of Theorem 1 applied to $C \backslash\{1, \ldots, c-3\}$ changing only preferences of agents $c-1$ and $c$ going from $R_{N} \equiv\left(\widetilde{R}_{\{1, \ldots, c-2\}}, R_{\{c-1, c\}}^{\prime}, R_{-C}\right)$ to $\left(\widetilde{R}_{C}, R_{-C}\right)$ and obtaining a contradiction that coalition $C \backslash\{1, \ldots, c-3\}$ manipulates $f$ at $\left(\widetilde{R}_{\{1, \ldots, c-3\}}, R_{C \backslash\{1, \ldots, c-3\}}^{\prime}, R_{-C}\right)$ via $\widetilde{R}_{C \backslash\{1, \ldots, c-3\}}$.

Case (c-1).2. If $R_{N}$ does not satisfy sequential inclusion, since the domain satisfies indirect sequential inclusion, by the above Remark applied to $y, z_{c-1}$ and $T=\{c-1, c\} \subset S\left(R_{N}\right.$; $\left.y, z_{c-1}\right)=S\left(\left(\widetilde{R}_{\{1, \ldots, c-2\}}, R_{C \backslash\{1, \ldots, c-2\}}^{\prime}, R_{-C}\right) ; y, z_{c-1}\right)$, there exists $R_{N}^{\prime}$ such that $R_{N \backslash\{c-1, c\}}^{\prime}=$ $R_{N \backslash\{c-1, c\}}$ such that conditions $\left(1^{\prime}\right),\left(2^{\prime}\right)$, and $\left(3^{\prime}\right)$ in the above Remark hold. By the above Lemma and $\left(1^{\prime}\right)$, we obtain that $f\left(R_{\{c-1, c\}}^{\prime}, R_{N \backslash\{c-1, c\}}\right)=z_{c-1}$. By condition $\left(2^{\prime}\right)$, for $c-1$ and $c, y=f\left(\widetilde{R}_{C}, R_{-C}\right) P_{i}^{\prime} f\left(R_{\{c-1, c\}}^{\prime}, R_{N \backslash\{c-1, c\}}\right)=z_{c-1}$. Thus, coalition $\{c-1, c\}$ manipulates $f$ at $\left(R_{\{c-1, c\}}^{\prime}, R_{N \backslash\{c-1, c\}}\right)=\left(\widetilde{R}_{\{1, \ldots, c-2\}}, R_{\{c-1, c\}}^{\prime}, R_{-C}\right)$ via $\widetilde{R}_{\{c-1, c\}}$. By $\left(3^{\prime}\right), \succsim\left(R_{N}^{\prime}\right.$; $\left.y, z_{c-1}\right)$ is complete and acyclic on $\{c-1, c\}$. Choose an agent in $C\left(\{c-1, c\}, \succsim\left(R_{N}^{\prime} ; y, z_{c-1}\right)\right)$, without loss of generality, say agent $c-1$. Then, $L\left(R_{c-1}^{\prime}, z_{c-1}\right) \subset \bar{L}\left(R_{h}^{\prime}, y\right)$ for $h=c$. Change preferences of agent $c-1$ and let $z_{c}=f\left(\widetilde{R}_{\{1, \ldots, c-1\}}, R_{c}^{\prime}, R_{-C}\right)$. By strategy-proofness, $z_{c} \in$ $L\left(R_{c-1}^{\prime}, z_{c-1}\right)$. Thus $z_{c} \in \bar{L}\left(R_{c}^{\prime}, y\right)$, which implies that $L\left(R_{c}^{\prime}, z_{c}\right) \subset \bar{L}\left(R_{c}^{\prime}, y\right)$. Go to Step c.

Step c: Let $z_{c+1}=f\left(\widetilde{R}_{C}, R_{-C}\right)$ (that is, $\left.z_{c+1}=y\right)$. By strategy-proofness and definition of $z_{c}$, $z_{c+1} \in L\left(R_{c}^{\prime}, z_{c}\right)$. Thus $z_{c+1}=y \in \bar{L}\left(R_{c}^{\prime}, y\right)$ which is the desired contradiction. This ends the proof of Theorem 2 . 
Next, we state the relationship between our Theorem 2 and Theorem 1 in Le Breton and Zaporozhets [12] for onto social choice functions.

Proof of Proposition 2. If for any $R_{N} \in \mathcal{R}^{n}, R_{N}$ satisfies sequential inclusion, the proof ends. Otherwise, let $R_{N} \in \mathcal{R}^{n}$ be a preference profile violating sequential inclusion. We will now check that $R_{N}$ satisfies condition (b) in Definition 8. Let $y, z \in A$ and define $R_{N}^{\prime}$ as follows: For any $i \in N \backslash S\left(R_{N} ; y, z\right)$, let $R_{i}^{\prime}=R_{i}$. Consider an agent $i \in S\left(R_{N} ; y, z\right)$. By regularity of $f$, there exists $\bar{R}_{i} \in \mathcal{R}$ such that the best alternative of $\bar{R}_{i}$ is $y$, since by ontoness, $y \in A_{f}$. Additionally, since $y P_{i} z$, by richness of the domain there exists $\widehat{R}_{i} \in \mathcal{R}$ such that $y$ is the best alternative according to $\widehat{P}_{i}$, and for all $x \neq z$ such that $z R_{i} x$, we have $z \widehat{P}_{i} x$. Thus, for any $i \in S\left(R_{N} ; y, z\right)$, let us define $R_{i}^{\prime}=\widehat{R}_{i}$. Clearly, by definition of $R_{N}^{\prime}$, conditions (1) and (2) in part (b) of Definition 8 are satisfied. Concerning condition (3), observe that for any $i, j \in S\left(R_{N} ; y, z\right), i \sim j$. Thus, $\succsim S\left(R_{N} ; y, z\right)$ is complete and acyclic which ends the proof.

Finally, we prove our necessity result.

Proof of Theorem 4. Our strategy of proof is as follows. Let $\times_{i \in N} \mathcal{R}_{i}$ be a domain where sequential inclusion is violated. We shall construct a subdomain and exhibit a rule on it which is strategy-proof but not group strategy-proof.

We will structure our argument as follows. We first present three lemmas, and then provide our construction as a final step.

We begin by Lemma 1 . Remember that sequential inclusion requires our binary relation $\succsim$ to be complete and acyclic for all pairs of alternatives $y, z$. Therefore, its violation can come from two sources. If $\succsim$ is incomplete on a pair $y, z$, then it is easy to show that there are four distinct alternatives and two preference relations in the domain satisfying enough properties to allow for our construction. The starting point for this reasoning is the following lemma.

Lemma 1. Let $R_{N} \in \times_{i \in N} \mathcal{R}_{i}$ and $y, z \in A$. If $\succsim\left(R_{N} ; y, z\right)$ on $S\left(R_{N} ; y, z\right)$ is not complete then there exist at least four alternatives in $A$, say $y, z, a_{1}$, and $a_{2}$. Moreover, there exist 2 individual preference relations in $R_{N}$, say $R^{1}, R^{2}$ such that $a_{1} R^{1} y, y P^{1} z, z R^{1} a_{2}$, and $a_{2} R^{2} y, y P^{2} z, z R^{2} a_{1}$.

Proof. For completeness to fail, there must exist two alternatives $y$ and $z$ and two individuals $i$ and $j$ such that $\neg\left[L\left(R_{i}, z\right) \subset \bar{L}\left(R_{j}, y\right)\right]$ and $\neg\left[L\left(R_{j}, z\right) \subset \bar{L}\left(R_{i}, y\right)\right]$ hold. There will also be $a_{1}, a_{2} \in A \backslash\{y, z\}$ such that $a_{2} \in L\left(R_{i}, z\right)$ but $a_{2} \notin \bar{L}\left(R_{j}, y\right)$ and $a_{1} \in L\left(R_{j}, z\right)$ but $a_{1} \notin \bar{L}\left(R_{i}, y\right)$ (note that $a_{1} \neq z$ since $z \in \bar{L}\left(R_{i}, y\right)$ but $a_{1} \notin \bar{L}\left(R_{i}, y\right)$. Similarly, $a_{1} \neq y$ since $a_{1} \in L\left(R_{j}, z\right)$ but $\left.y \notin L\left(R_{j}, z\right)\right)$. Finally, observe that $a_{1} \neq a_{2}$ : since $a_{1} \in L\left(R_{j}, z\right)$ and $y P_{j} z$ then $a_{1} \in \bar{L}\left(R_{j}, y\right)$. But, $a_{2} \notin \bar{L}\left(R_{j}, y\right)$. We have then shown that the violation of completeness requires at least four distinct alternatives. Furthermore, observe that the preferences of the two agents $i$ and $j$ satisfy the conditions predicated by the lemma. Then, define $R^{1}=R_{i}$ and $R^{2}=R_{j}$. This ends the proof of Lemma 1.

Lemma 1 identifies the alternatives and the preferences on them that will help us determine a subdomain where equivalence will not hold, given that $\succsim$ is not acyclic. This construction of such a subdomain and of a function that is strategy-proof on it, but group manipulable (in fact, by only two-agent coalitions) is the essence of the proof of Lemma 3.

We now turn to the case where $\succsim$ is complete for all pairs and all profiles, and the violation of sequential inclusion arises because it is cyclical for some profile and some pair. We concen- 
Table VI

The four preferences over six alternatives in Example 5 (see proof of Theorem 4).

\begin{tabular}{llll}
\hline$R^{1}$ & $R^{2}$ & $R^{3}$ & $R^{4}$ \\
\hline$a_{1}$ & $a_{2}$ & $a_{3}$ & $a_{4}$ \\
$y$ & $y$ & $y$ & $y$ \\
$a_{2}$ & $a_{3}$ & $a_{4}$ & $a_{1}$ \\
$a_{3}$ & $a_{1}$ & $a_{2}$ \\
$z$ & $a_{4}$ & $z$ & $z$ \\
$a_{4}$ & $z$ & $a_{2}$ & $a_{3}$ \\
\hline
\end{tabular}

trate on one cycle of minimal length $k$, and prove that its existence requires that a number of distinct preferences on at least $k+2$ distinct alternatives exist in the initial domain. We shall use these preferences to construct a subdomain and to define a social choice function on it that is strategy-proof but not group strategy-proof. The starting point for the construction is provided by Lemma 2.

Lemma 2. Let $R_{N} \in \times_{i \in N} \mathcal{R}_{i}$ and $y, z \in A$ such that $\succsim\left(R_{N} ; y, z\right)$ on $S\left(R_{N} ; y, z\right)$ has a cycle of size $k, 3 \leqslant k \leqslant s$ but $\succsim\left(R_{N} ; y, z\right)$ does not have any cycle of lower size. Then, there exist at least $k+2$ alternatives in $A$, say $y, z, a_{1}, a_{2}, \ldots, a_{k} \in A$. There also exist $k$ individual preference relations in $R_{N}$, say $R^{1}, R^{2}, \ldots, R^{k}$, such that for any $l=1, \ldots, k \in N$, the following holds: [(1) $a_{l} R^{l} y$, (2) $z R^{l} a_{l-1}$, for any $j=1, \ldots, k-2$, (3) $y P^{l} a_{l+j}$, and (4) $a_{l+j} P^{l} z$ ]. Moreover, if for any alternative $w \in A$, the binary relation $\succsim\left(R_{N} ; y, w\right)$ on $S\left(R_{N} ; y, w\right)$ is complete, then (5) for any $l=1, \ldots, k,\left[a_{l+j} P^{l} a_{l+1+j}\right.$, for $\left.j=1, \ldots, k-3\right] .^{8}$

Again, Lemma 2 allows us to identify alternatives and specific preferences that will be used to construct a subdomain where to define a function that proves the lack of equivalence.

Before turning to the proof we introduce a piece of notation and we present an example of the type of reasoning that we shall be using.

Concerning notation, we define the following equivalence relation on the set of integers. Let $k \in \mathbb{Z}$ ( $\mathbb{Z}$ is the set of integers), $k \leqslant n$, and $i, j \in \mathbb{Z}$ we say that $j$ and $i$ are equivalent modulo $k$, say $j \equiv i(\bmod \mathrm{k})$, if either $j=i$ or else $j=i+v k$, for some $v \in \mathbb{Z}$.

In what follows, whenever we have $j \equiv i(\bmod \mathrm{k})$, then we will write $R_{j}=R_{i}$. We will do the same and $R^{j}=R^{i}$, when we refer to a list of specific preference relations labelled by their superindex, or $a_{j}=a_{i}$, where these are the subindices in a list of alternatives.

Now, Example 3 shows how, given a profile that generates a cycle in our binary relation $\succsim$, this allows us to single out some set of preferences that will later be used to construct the domain we are looking for. The argument in the proof of Lemma 2 extends the intuition in this example.

Example 3. Take $N=\{1,2,3,4,5\}$ and $A=\left\{a_{1}, a_{2}, a_{3}, a_{4}, a_{5}, y, z\right\}$. Let $R_{N} \in \times_{i \in N} \mathcal{R}_{i}$ such that $\succsim\left(R_{N} ; y, z\right)$ on $S\left(R_{N} ; y, z\right)$ has a cycle of size four, but none of lower size. Note that this means that $\# S\left(R_{N} ; y, z\right) \geqslant 4$. We single out four preference relations in $R_{N}$ and six alternatives in $A$ in such a way that those preferences, when restricted to the six alternatives, are as in Table VI.

Note that we do not say anything about the relative position of $a_{5}$ with respect to any other alternative: it could be any. Observe that the relation $\succsim\left(R_{N} ; y, z\right)$ over agents having the prefer-

\footnotetext{
8 The latter condition has some bite only for $k \geqslant 4$.
} 
ences in the table has a cycle of size four, is complete and has no cycle of size 3 . In particular, observe that the following binary relations hold: $1 \succ 2,2 \succ 3,3 \succ 4$ and $4 \succ 1$. Moreover, $1 \sim 3$, $2 \sim 4$.

Proof of Lemma 2. We first prove that there must exist alternatives and individuals whose preferences satisfy (1) and (2). Without loss of generality, let the cycle of $k$ agents be $1 \succ 2 \succ 3 \succ$ $\cdots \succ k$ and $k \succ 1$. Thus, their individual preferences in $R_{N}$, say $R_{1}, R_{2}, \ldots, R_{k}$, are such that $L\left(R_{l}, z\right) \subset \bar{L}\left(R_{l+1}, y\right)$ and $\neg\left[L\left(R_{l+1}, z\right) \subset \bar{L}\left(R_{l}, y\right)\right]$ for $l=1, \ldots, k$. From the latter expression, for any $l=1, \ldots, k$, there exists $a_{l} \in A \backslash\{z, y\}$ such that $z R_{l+1} a_{l}$ and $a_{l} R_{l} y$. Note that for any $l=1, \ldots, k, a_{l} \neq z$ and $a_{l} \neq y$ since $y P_{l} z$ and $y P_{l+1} z$, respectively. Thus, there exist $k$ individual preference relations in $R_{N}$, in particular: $R_{1}, R_{2}, \ldots, R_{k}$, such that expressions (1) and (2) in the lemma hold. We now show that the alternatives $a_{l}$ so defined are all distinct.

For any $i, 1 \leqslant i \leqslant k-1,\left[a_{i} \neq a_{j}\right.$, for any $\left.j, k \geqslant j>i\right]$.

Let $i, j$ such that $j-i=1$. Given the above cycle of $k$ agents, $j \succ j+1$, thus $a_{j-1} \in$ $L\left(R_{j}, z\right) \subset \bar{L}\left(R_{j} y\right)$. But we also proved that $a_{j} \notin \bar{L}\left(R_{j}, y\right)$. Thus, $a_{j} \neq a_{j-1}$.

Let $i, j$ such that $j-i=2$. Given the above cycle of $k$ agents, $j-1 \succ j$, thus $a_{j-2} \in$ $L\left(R_{j-1}, z\right) \subset \bar{L}\left(R_{j}, y\right)$. But since $j \succ j+1$, by $a_{j} \notin \bar{L}\left(R_{j}, y\right)$. Thus, $a_{j} \neq a_{j-1}$.

Let $i, j$ such that $j-i=t$, for $3 \leqslant t \leqslant k-1$. By assumption, there is no cycle of agents in $S\left(R_{N} ; y, z\right)$ of size $t$. Thus, given the above cycle of $k$ agents, $i+1 \succ i+2 \succ \cdots \succ i+(t-1) \succ j$ and $i+1 \succsim j$. Thus, $a_{i} \in L\left(R_{i+1}, z\right) \subset \bar{L}\left(R_{j}, y\right)$. But we also proved that $a_{j} \notin \bar{L}\left(R_{j}, y\right)$. Thus, $a_{i} \neq a_{j}$.

We now prove that the preferences in question satisfy expression (3).

For any $l=1, \ldots, k,\left[a_{l+j} P_{l} z\right.$ for any $\left.j=1, \ldots, k-2\right]$.

Take any $j$. By assumption, there is no cycle of agents in $S\left(R_{N} ; y, z\right)$ of size $j+1$ (since $2 \leqslant j+1 \leqslant k-1)$. Thus, given the above defined cycle of $k$ agents, $l \succ l+1 \succ \cdots \succ l+j \succ j$ and $l \succsim l+j$. Thus, $L\left(R_{l}, z\right) \subset \bar{L}\left(R_{l+j}, y\right)$. If $z R_{l} a_{l+j}$ then $a_{l+j} \in \bar{L}\left(R_{l+j}, y\right)$ which contradicts expression (1) above proved.

We now show that the preferences also satisfy expression (4).

For any $l=1, \ldots, k,\left[y P_{l} a_{l+j}\right.$ for any $\left.j=1, \ldots, k-2\right]$.

Take any $j$. By assumption, there is no cycle of agents in $S\left(R_{N} ; y, z\right)$ of size $k-j$ (since $2 \leqslant k-j \leqslant k-1)$. Thus, given the above defined cycle of $k$ agents $l-(k-j-1) \succ l-(k-j) \succ$ $l-(k-j+1) \succ \cdots \succ l-3 \succ l-2 \succ l-1 \succ l$ and $l-(k-j-1) \succsim l$. Using our equivalence, $l+j+1 \equiv l+j+1-k(\bmod \mathrm{k})$ and thus $R_{l+j+1}=R_{l+j+1-k}$. Thus, $L\left(R_{l+j+1}, z\right) \subset \bar{L}\left(R_{l}, y\right)$. By the previous lemma, we know that $a_{l+j} \in L\left(R_{l+j+1}, z\right)$, and thus $a_{l+j} \in \bar{L}\left(R_{l}, y\right)$.

For $k=3$ the lemma is proved.

For $k \geqslant 4$, we finally prove that the preferences also satisfy expression (5).

For any $l=1, \ldots, k,\left[a_{l+j} P_{l} a_{l+1+j}\right.$, for $\left.j=1, \ldots, k-3\right]$.

We first show that for any $l=1, \ldots, k, a_{l+1} P_{l} a_{l+1+j}$ for $j=1, \ldots, k-3$. (*)

Fix $l$ and $j$. By contradiction, suppose that $a_{l+1+j} R_{l} a_{l+1}$. Consider the pair of alternatives $\left(y, a_{l+1+j}\right)$. By hypothesis, the binary relation $\succsim\left(R_{N} ; y, a_{l+1+j}\right)$ on $S\left(R_{N} ; y, a_{l+1+j}\right)$ is complete. Since $l, l+1 \in S\left(R_{N} ; y, a_{l+1+j}\right)$, either $l \succsim l+1$ or $l+1 \succsim l$ should hold. By hypothesis, $a_{l+1} \in L\left(R_{l}, a_{l+1+j}\right)$ and, by conditions (1) to (4) already proved, $a_{l+1} \notin \bar{L}\left(R_{l+1}, y\right)$. Then, $\neg\left[L\left(R_{l}, a_{l+1+j}\right) \subset \bar{L}\left(R_{l+1}, y\right)\right]$, or equivalently, $\neg[l \succsim l+1]$. On the other hand, by conditions (1) to (4), $a_{l} \notin \bar{L}\left(R_{l}, y\right)$, but also $a_{l} \in L\left(R_{l+1}, z\right)$ and $a_{l+1+j} P_{l+1} z$ holds. The latter two expressions imply that $a_{l} \in L\left(R_{l+1}, a_{l+1+j}\right)$. Then, $\neg\left[L\left(R_{l+1}, a_{l+1+j}\right) \subset \bar{L}\left(R_{l}, y\right)\right]$, or equivalently, $\neg[l+1 \succsim l]$ which is the desired contradiction. 
For $k=4$ the lemma is proved.

Now, for $k>4$, we show that for any $l=1, \ldots, k, a_{l+t} P_{l} a_{l+t+1}$ for any $t, t=2, \ldots, k-3$.

Fix $l$ and $t$. By contradiction, suppose that $a_{l+t+1} R_{l} a_{l+t}$. Consider the pair of alternatives $\left(y, a_{l+t+1}\right)$. By hypothesis, the binary relation $\succsim\left(R_{N} ; y, a_{l+t+1}\right)$ on $S\left(R_{N} ; y, a_{l+t+1}\right)$ is complete. Since $l, l+t \in S\left(R_{N} ; y, a_{l+t+1}\right)$, either $l \succsim l+t$ or $l+t \succsim l$ should hold. By hypothesis, $a_{l+t} \in L\left(R_{l}, a_{l+t+1}\right)$ and, by conditions (1) to (4), $a_{l+t} \notin \bar{L}\left(R_{l+t}, y\right)$. Then, $\neg\left[L\left(R_{l}, a_{l+t+1}\right) \subset \bar{L}\left(R_{l+t}, y\right)\right]$, or equivalently, $\neg[l \succsim l+t]$. On the other hand, by conditions (1) to $(4), a_{l} \notin \bar{L}\left(R_{l}, y\right)$. Using the modulo $k$ equivalence, and by the previous statement $(*)$ applied for $j=k-1-t$ and for $l \equiv l+t, a_{l+t+1} P_{l+t} a_{l}$ (that is, $a_{l} \in L\left(R_{l+t}, a_{l+t+1}\right)$ ). Then, $\neg\left[L\left(R_{l+t}, a_{l+t+1}\right) \subset \bar{L}\left(R_{l}, y\right)\right]$, or equivalently, $\neg[l+t \succsim l]$ which is the desired contradiction.

Observe that the preferences of the $k$ agents, $1, \ldots, k$, satisfy the conditions predicated by the lemma. Again define $R^{i}=R_{i}$ for all $i=1, \ldots, k$. This ends the proof of Lemma 2.

Next we prove a lemma that already establishes our result for the case where sequential inclusion is violated by the lack of completeness of our binary relation. ${ }^{9}$ Let first define pairwise strategy-proofness, mainly no coalition of 1 or 2 agents can manipulate the rule at any profile.

Definition. A social choice function $f$ is pairwise strategy-proof on $\times_{i \in N} \mathcal{R}_{i}$ if $f$ is not group manipulable on $\times_{i \in N} \mathcal{R}_{i}$ for any $R_{N} \in \times_{i \in N} \mathcal{R}_{i}$ by any coalition $C \subset N$ with $\# C \leqslant 2$.

Lemma 3. Let $\times_{i \in N} \mathcal{R}_{i}$ be a domain on which any strategy-proof social choice function on $\times_{i \in N} \mathcal{D}_{i} \subset \times_{i \in N} \mathcal{R}_{i}$ is also pairwise strategy-proof on $\times_{i \in N} \mathcal{D}_{i}$. Then, for any profile $R_{N} \in$ $\times_{i \in N} \mathcal{R}_{i}$ and for any pair $y, z \in A, \succsim\left(R_{N} ; y, z\right)$ on $S\left(R_{N} ; y, z\right)$ is complete.

Proof. Suppose that there exists $R_{N} \in \times_{i \in N} \mathcal{R}_{i}$ and a pair of alternatives $y, z \in A$, such that the binary relation $\succsim\left(R_{N} ; y, z\right)$ defined on $S\left(R_{N} ; y, z\right)$ is not complete.

Therefore there exists a pair of agents $i, j \in S\left(R_{N} ; y, z\right)$ such that neither $i \succsim j$ nor $j \succsim$ $i$. By Lemma 1 we know that there exist $a_{1}, a_{2} \in A \backslash\{y, z\}$ and $a_{1} \neq a_{2}$, and there exist two individual preference relations in $R_{N}$, say $R^{1}, R^{2}$ such that $a_{1} R^{1} y, y P^{1} z, z R^{1} a_{2}$, and $a_{2} R^{2} y$, $y P^{2} z, z R^{2} a_{1}$. Define $\mathcal{D}_{i}=\left\{R^{1}, \bar{R}^{1}\right\}, \mathcal{D}_{j}=\left\{R^{2}, \bar{R}^{2}\right\}$ for $i, j, \mathcal{D}_{l}=\mathcal{R}_{l}$ for any $l \in N \backslash\{i, j\}$. Note that $\bar{R}^{1}$ and $\bar{R}^{2}$ are the opposite preferences of $R^{1}$ and $R^{2}$, respectively. Now construct $f$ as follows: for any $\widehat{R}_{-\{i, j\}} \in \times_{l \in N \backslash\{i, j\}} \mathcal{R}_{l}, f\left(R_{i}^{1}, \bar{R}_{j}^{2} ; \widehat{R}_{-\{i, j\}}\right)=a_{1}, f\left(\bar{R}_{i}^{1}, R_{j}^{2} ; \widehat{R}_{-\{i, j\}}\right)=a_{2}$, $f\left(R_{i}^{1}, R_{j}^{2} ; \widehat{R}_{-\{i, j\}}\right)=z, f\left(\bar{R}_{i}^{1}, \bar{R}_{j}^{2} ; \widehat{R}_{-\{i, j\}}\right)=y$. Note that $f$ is strategy-proof but not pairwise strategy-proof. This ends the proof of Lemma 3.

\section{A final step: identifying a domain and defining a rule.}

Suppose, without loss of generality, that $\times_{i \in N} \mathcal{R}_{i}$ is a domain such that for any $R_{N} \in \times_{i \in N} \mathcal{R}_{i}$ and any pair of alternatives $y, z \in A, \succsim\left(R_{N} ; y, z\right)$ on $S\left(R_{N} ; y, z\right)$ is complete but sequential inclusion does not hold. Then, there exists $\widetilde{R}_{N} \in \times_{i \in N_{\widetilde{P}}} \mathcal{R}_{i}$, a pair of alternatives $y, z \in A$, such that $\succsim\left(\widetilde{R}_{N} ; y, z\right)$ defined on $S\left(\widetilde{R}_{N} ; y, z\right)=\left\{i \in N: y \widetilde{P}_{i} z\right\}$ has cycles. Let $k \geqslant 3$ be the minimal size of these cycles. Therefore there exists a set of $k$ agents, say $S_{k}=\{1,2, \ldots, k\}$ included in $S\left(\widetilde{R}_{N} ; y, z\right)$ such that $1 \succ 2 \succ \cdots \succ k$ and $k \succ 1$. Thus, there exist at least $k+2$ alternatives, say

9 This is the first instance where we use the assumption that the domain admits opposite preferences. Lemmas 1 and 2 do not require it and could therefore be used to explore similar partial necessity results on domains satisfying alternative requirements. 
Table VII

Borda $^{\varepsilon}$ scores in the definition of the rule in the proof of Theorem 4.

\begin{tabular}{|c|c|c|c|c|c|c|c|}
\hline$a_{i} \backslash R^{j}$ & $R^{1}$ & $R^{2}$ & $R^{3}$ & $\ldots$ & $R^{k-2}$ & $R^{k-1}$ & $R^{k}$ \\
\hline $\operatorname{Borda}^{\varepsilon}\left(R^{j} ; a_{1}\right)$ & $k-1+\varepsilon$ & 0 & 1 & & $k-4$ & $k-3$ & $k-2+\varepsilon$ \\
\hline $\operatorname{Borda}^{\varepsilon}\left(R^{j} ; a_{2}\right)$ & $k-2+\varepsilon$ & $k-1+\varepsilon$ & 0 & & $k-5$ & $k-4$ & $k-3+\varepsilon$ \\
\hline $\operatorname{Borda}^{\varepsilon}\left(R^{j} ; a_{3}\right)$ & $k-3+\varepsilon$ & $k-2+\varepsilon$ & $k-1+\varepsilon$ & & $k-6$ & $k-5$ & $k-4+\varepsilon$ \\
\hline$\ldots$ & & & & & & & \\
\hline $\operatorname{Borda}^{\varepsilon}\left(R^{j} ; a_{k-2}\right)$ & $2+\varepsilon$ & $3+\varepsilon$ & $4+\varepsilon$ & & $k-1+\varepsilon$ & 0 & $1+\varepsilon$ \\
\hline $\operatorname{Borda}^{\varepsilon}\left(R^{j} ; a_{k-1}\right)$ & $1+\varepsilon$ & $2+\varepsilon$ & $3+\varepsilon$ & & $k-2+\varepsilon$ & $k-1+\varepsilon$ & 0 \\
\hline $\operatorname{Borda}^{\varepsilon}\left(R^{j} ; a_{k}\right)$ & 0 & 1 & 2 & & $k-3$ & $k-2$ & $k-1+\varepsilon$ \\
\hline
\end{tabular}

$y, z, a_{1}, a_{2}, \ldots, a_{k} \in A$, and $k$ individual preference relations in $\widetilde{R}_{N}$, say $R^{1}, \ldots, R^{k}$, satisfying conditions (1) to (5) in Lemma 2.

We now identify a subdomain and a rule on it that is strategy-proof but not group strategyproof.

Each individual will have two possible preferences. Then, out of all possible profiles we will single out two of them: the one where each agent $i$ has preferences $R^{i}$, and the one where each agent $i$ has opposite preferences $\bar{R}^{i}$. All other profiles will be classified in a way that depends on the sets of agents that have preferences of one type and of the other. Formally, consider the following subdomain of $\times_{i \in N} \mathcal{R}_{i}$. For any $l \in N \backslash\{1, \ldots, k\}$, define $\mathcal{D}_{l}=\mathcal{R}_{l}$. For any $i=$ $1, \ldots, k$, define $\mathcal{D}_{i}=\left\{R^{i}, \bar{R}^{i}\right\}$ where $\bar{R}^{i}$ is the opposite preference of $R^{i}$. Clearly, $\times_{i \in N} \mathcal{D}_{i} \subset$ $\times_{i \in N} \mathcal{R}_{i}$. Let $\widetilde{R}_{S_{k}}=\left(R^{1}, \ldots, R^{k}\right)$ and call $\bar{R}_{S_{k}}=\left(\bar{R}^{1}, \ldots, \bar{R}^{k}\right)$.

Let $R_{S_{k}} \in \times_{i \in S_{k}} \mathcal{D}_{i}$. We define a partition of $S_{k}$ that depends on $R_{S_{k}}$ : Let $S_{k, z}\left(R_{S_{k}}\right)=\left\{i \in S_{k}\right.$ : $\left.R_{i} \neq R^{i}\right\}$ and $S_{k, y}\left(R_{S_{k}}\right)=\left\{j \in S_{k}: R_{j}=R^{j}\right\}$.

Now construct $f$ as follows: for any $\widehat{R}_{-S_{k}} \in \times_{l \in N \backslash S_{k}} \mathcal{D}_{l}$,

$$
\begin{aligned}
& f\left(R_{1}^{1}, \ldots, R_{k}^{k}, \widehat{R}_{-S_{k}}\right)=f\left(\widetilde{R}_{S_{k}}, \widehat{R}_{-S_{k}}\right)=z, \\
& f\left(R_{1}^{1+t}, \ldots, R_{k}^{k+t}, \widehat{R}_{-S_{k}}\right)=f\left(\bar{R}_{S_{k}}, \widehat{R}_{-S_{k}}\right)=y, \quad \text { and } \\
& f\left(R_{S_{k}}, \widehat{R}_{-S_{k}}\right)=\arg \max _{a_{l} \in\left\{a_{1}, \ldots, a_{k}\right\}} \operatorname{Borda}^{\varepsilon}\left(\left(R_{S_{k}} ; \widehat{R}_{-S_{k}}\right) ; a_{l}\right), \quad \text { for any other } R_{S_{k}} \in \times_{i \in S_{k}} \mathcal{D}_{i},
\end{aligned}
$$

where $\operatorname{Borda}^{\varepsilon}\left(\left(R_{S_{k}} ; \widehat{R}_{-S_{k}}\right) ; a_{l}\right) \equiv \sum_{j \in S_{k, y}\left(R_{S_{k}}\right)} \operatorname{Borda}^{\varepsilon}\left(R_{j} ; a_{l}\right)$, with $\operatorname{Borda}^{\varepsilon}\left(R_{j} ; a_{l}\right)$ the score that alternative $a_{l}$ receives under individual preference $R_{j}$ for $j \in S_{k, y}\left(R_{S_{k}}\right)$ as given in Table VII and with $\varepsilon>0$ and $\varepsilon<\frac{1}{k-2}$.

Notice first that only agents in $S_{k, y}$ play an effective role in the social choice function. The rest are dummies. Next, we should explain what we mean by the expression above: $\operatorname{Bor} d a^{\varepsilon}(\cdot)$. What we do is to compute a variant of the Borda count for all those profiles that are different from the two we singled out to produce $z$ and $y$, respectively. In this version of the Borda count, each alternative receives the points that correspond to it because of its position, plus eventually some value $\varepsilon$. The alternatives and positions that get this extra epsilon are chosen in such a way that no ties arise among winners within the domain. Therefore, we are defining a function, not a correspondence. The specific values that apply under this rule for our profiles are given in Table VII.

Note that for any $\widehat{R}_{-S_{k}} \in \times_{l \in N \backslash S_{k}} \mathcal{R}_{l}$ and any $i \in\{1, \ldots, k\}, f\left(\widehat{R}_{S_{k}}, \widehat{R}_{-S_{k}}\right)=a_{i}$, where $\widehat{R}_{S_{k}} \in$ $\times_{l \in S_{k}} \mathcal{D}_{l} \backslash\left\{\widetilde{R}_{S_{k}}, \bar{R}_{S_{k}}\right\}$ is such that $S_{k, y}\left(\widehat{R}_{S_{k}}\right)=\{i\}$. Thus, the range of the social choice function is $A_{f}=\left\{a_{1}, \ldots, a_{k}, y, z\right\}$. 
We now show the following two claims for any $R_{S_{k}} \in \times_{l \in S_{k}} \mathcal{D}_{l} \backslash\left\{\widetilde{R}_{S_{k}}, \bar{R}_{S_{k}}\right\}$, and $\widehat{R}_{-S_{k}} \in$ $\times_{l \in N \backslash S_{k}} \mathcal{R}_{l}$. Note that $S_{k, z}\left(R_{S_{k}}\right)$ and $S_{k, y}\left(R_{S_{k}}\right)$ are non-empty sets. In words, Claim 1 requires that at least one agent belonging to the set $S_{k, y}$, of agents $i$ with preference $R^{i}$, has no incentive to deviate to alternative $y$. Claim 2 requires that at least one agent belonging to the set $S_{k, z}$, of agents $i$ with preference $R^{i \prime}$, has no incentive to deviate from alternative $z$.

Claim 1. Let $R_{S_{k}} \in \times_{l \in S_{k}} \mathcal{D}_{l} \backslash\left\{\widetilde{R}_{S_{k}}, \bar{R}_{S_{k}}\right\}$ and $\widehat{R}_{-S_{k}} \in \times_{l \in N \backslash S_{k}} \mathcal{R}_{l}$. Then,

$$
f\left(R_{S_{k}}, \widehat{R}_{-S_{k}}\right) \in \bigcup_{j \in S_{k, y}\left(R_{S_{k}}\right)} U\left(R_{j}, y\right),
$$

where $U\left(R_{j}, y\right)=\left\{x \in A: x R_{j} y\right\}$.

Proof. By contradiction, suppose that, without loss of generality, $f\left(R_{S_{k}}, \widehat{R}_{-S_{k}}\right)=a_{i}$ such that $y P_{j} a_{i}$ for all $j \in S_{k, y}\left(R_{S_{k}}\right)$. Thus, $i \notin S_{k, y}\left(R_{S_{k}}\right)$. Therefore,

$$
\sum_{j \in S_{k, y}\left(R_{S_{k}}\right)} \operatorname{Borda}^{\varepsilon}\left(R_{j} ; a_{(i-1) \bmod k}\right)>\sum_{j \in S_{k, y}\left(R_{S_{k}}\right)} \operatorname{Borda}^{\varepsilon}\left(R_{j} ; a_{i}\right),
$$

and thus $f\left(R_{S_{k}}, \widehat{R}_{-S_{k}}\right) \neq a_{i}$ which is the desired contradiction. This ends the proof of Claim 1.

Claim 2. Let $R_{S_{k}} \in \times_{l \in S_{k}} \mathcal{D}_{l} \backslash\left\{\widetilde{R}_{S_{k}}, \bar{R}_{S_{k}}\right\}$ and $\widehat{R}_{-S_{k}} \in \times_{l \in N \backslash S_{k}} \mathcal{R}_{l}$. Then,

$$
f\left(R_{S_{k}}, \widehat{R}_{-S_{k}}\right) \in \bigcup_{i \in S_{k, z}\left(R_{S_{k}}\right)} L\left(R_{i}, z\right) .
$$

Proof. By contradiction, suppose that, without loss of generality, $f\left(R_{S_{k}}, \widehat{R}_{-S_{k}}\right)=a_{l}$ such that $a_{l} \notin \bigcup_{i \in S_{k, z}\left(R_{S_{k}}\right)} L\left(R_{i}, z\right)$. Therefore, $(l+1) \bmod k \in S_{k, y}\left(R_{S_{k}}\right)$. For notational simplicity, we will write $(l+1)$ instead. By Claim $1, l \in S_{k, y}\left(R_{S_{k}}\right)$. Note that for any $l=1, \ldots, k$,

$$
\operatorname{Borda}^{\varepsilon}\left(R_{l+1} ; a_{l+1}\right)-\operatorname{Borda}^{\varepsilon}\left(R_{l+1} ; a_{l}\right)=k-1+\varepsilon .
$$

Since $S_{k, z}\left(R_{S_{k}}\right)$ is not empty, if $l \neq k-1$ we have

$$
\sum_{j \in S_{k, y}\left(R_{S_{k}}\right) \backslash\{l+1\}} \operatorname{Borda}^{\varepsilon}\left(R_{j} ; a_{l}\right)-\sum_{j \in S_{k, y}\left(R_{S_{k}}\right) \backslash\{l+1\}} \operatorname{Borda}^{\varepsilon}\left(R_{j} ; a_{l+1}\right)<k-1 .
$$

Then,

$$
\operatorname{Borda}^{\varepsilon}\left(\left(R_{S_{k}} ; \widehat{R}_{-S_{k}}\right) ; a_{l+1}\right)-\operatorname{Borda}^{\varepsilon}\left(\left(R_{S_{k}} ; \widehat{R}_{-S_{k}}\right) ; a_{l}\right)>k-1+\varepsilon+1-k=\varepsilon>0,
$$

and thus $f\left(R_{S_{k}}, \widehat{R}_{-S_{k}}\right) \neq a_{l}$ which is the desired contradiction.

If $l=k-1$ and since $S_{k, z}\left(R_{S_{k}}\right)$ is not empty,

$$
\sum_{j \in S_{k, y}\left(R_{S_{k}}\right) \backslash\{k\}} \operatorname{Borda}^{\varepsilon}\left(R_{j} ; a_{k-1}\right)-\sum_{j \in S_{k, y}\left(R_{S_{k}}\right) \backslash\{k\}} \operatorname{Borda}^{\varepsilon}\left(R_{j} ; a_{k}\right) \leqslant(k-2)(1+\varepsilon) .
$$

Therefore,

$$
\operatorname{Borda}^{\varepsilon}\left(\left(R_{S_{k}} ; \widehat{R}_{-S_{k}}\right) ; a_{k}\right)-\operatorname{Borda}^{\varepsilon}\left(\left(R_{S_{k}} ; \widehat{R}_{-S_{k}}\right) ; a_{k-1}\right) \geqslant 1-(k-3) \varepsilon
$$


where $1-(k-3) \varepsilon>0$ since $\varepsilon<\frac{1}{k-2}$. Thus $f\left(R_{S_{k}}, \widehat{R}_{-S_{k}}\right) \neq a_{l}=a_{k-1}$ which is the desired contradiction. This ends the proof of Claim 2.

We now show that $f$ is strategy-proof but it is not group strategy-proof. Observe that by construction of $f$, the only relevant deviating coalitions are those $C \subset S_{k}$. Since each agent in coalition $S_{k}$ strictly gains by deviating from $f\left(R_{1}^{1}, R_{2}^{2}, \ldots, R_{k}^{k}, \widehat{R}_{-S_{k}}\right)=z$ to $f\left(\bar{R}_{1}^{1}, \bar{R}_{2}^{2}, \ldots, \bar{R}_{k}^{k}\right.$, $\left.\widehat{R}_{-S_{k}}\right)=y$, then $f$ is not group strategy-proof.

We now check that no single individual $l \in S_{k}$ can manipulate $f$ at any profile. Let $\left(R_{S_{k}} ; \widehat{R}_{-S_{k}}\right) \in \times_{i \in N} \mathcal{D}_{i}$ be any profile. Remember that for any $l \in S_{k}, \mathcal{D}_{l}=\left\{R^{l}, \bar{R}^{l}\right\}$. We distinguish two cases:

Case 1. If $l \in S_{k, y}\left(R_{S_{k}}\right)$. By definition of $f, f\left(R_{S_{k}}, \widehat{R}_{-S_{k}}\right) \neq y$. Suppose that $f\left(R_{S_{k}}, \widehat{R}_{-S_{k}}\right)=a_{i}$ for some $i \in S_{k}$ and thus $S_{k, y}\left(R_{S_{k}}\right) \varsubsetneqq S_{k} \backslash\{l\}$. If agent $l$ announces $\bar{R}_{l}^{l}$ instead of $R_{l}^{l}$, in the definition of $f$ agent $l$ deducts more score to those alternatives he likes most. Therefore either the initial alternative $a_{i}$ or else a less preferred alternative for agent $l$ is chosen and agent $l$ does not manipulate $f$ at $\left(R_{S_{k}}, \widehat{R}_{-S_{k}}\right)$ via $\bar{R}^{l}$. An identical argument would apply if $f\left(R_{S_{k}}, \widehat{R}_{-S_{k}}\right)=z$ instead of $a_{i}$. This shows that no agent $l \in S_{k, y}\left(R_{S_{k}}\right)$ can manipulate $f$ at any profile $\left(R_{S_{k}} ; \widehat{R}_{-S_{k}}\right)$ via $\bar{R}_{l}^{l}$.

Case 2. If $l \in S_{k, z}\left(R_{S_{k}}\right)$. A similar argument to Case 1 works: By definition of $f, f\left(R_{S_{k}}, \widehat{R}_{-S_{k}}\right) \neq$ $z$. Suppose that $f\left(R_{S_{k}}, \widehat{R}_{-S_{k}}\right)=a_{i}$ for some $i \in S_{k}$ and thus $S_{k, y}\left(R_{S_{k}}\right) \subset S_{k} \backslash\{l\}$. If agent $l$ announces $R_{l}^{l}$ instead of $\bar{R}_{l}^{l}$, in the definition of $f$ agent $l$ adds more score to those alternatives he likes less (when announcing $R_{l}^{l}$ agent $l \in S_{k, y}\left(R_{l}^{l}, R_{S_{k} \backslash\{l\}} ; \widehat{R}_{-S_{k}}\right)$ ). Therefore either the initial alternative $a_{i}$ or else a less preferred alternative for agent $l$ is chosen and agent $l$ does not manipulate $f$ at $\left(R_{S_{k}}, \widehat{R}_{-S_{k}}\right)$ via $R^{l}$. An identical argument would apply if $f\left(R_{S_{k}}, \widehat{R}_{-S_{k}}\right)=y$ instead of $a_{i}$. This shows that no agent $l \in S_{k, y}\left(R_{S_{k}}\right)$ can manipulate $f$ at any profile $\left(R_{S_{k}} ; \widehat{R}_{-S_{k}}\right)$ via $R_{l}^{l}$.

\section{References}

[1] S. Barberà, A note on group strategy-proof decision schemes, Econometrica 47 (1979) 637-640.

[2] S. Barberà, M. Jackson, Strategy-proof exchange, Econometrica 63 (1995) 51-87.

[3] S. Barberà, H. Sonnenschein, L. Zhou, Voting by committees, Econometrica 59 (1991) 595-609.

[4] K. Border, J. Jordan, Straightforward elections, unanimity and phantom voters, Rev. Econ. Stud. 50 (1983) $153-170$.

[5] P. Dasgupta, P. Hammond, E. Maskin, The implementation of social choice rules: Some general results on incentive compatibility, Rev. Econ. Stud. 46 (1979) 185-216.

[6] A. Gibbard, Manipulation of voting schemes: A general result, Econometrica 41 (1973) 587-601.

[7] J. Green, J.-J. Laffont, On coalition incentive compatibility, Rev. Econ. Stud. 46 (1979) 243-254.

[8] K.H. Kim, F.W. Roush, Special domains and nonmanipulability, Math. Soc. Sci. 1 (1980) 85-92.

[9] B. Klaus, Coalitional strategy-proofness in economies with single-dipped preferences and the assignment of an indivisible object, Games Econ. Behav. 34 (2001) 64-82.

[10] M. Le Breton, A. Sen, Separable preferences, decomposability and strategyproofness, Econometrica 67 (1999) $605-628$.

[11] M. Le Breton, J. Weymark, Strategy-proof social choice with continuous separable preferences, J. Math. Econ. 32 (1999) 47-85.

[12] M. Le Breton, V. Zaporozhets, On the equivalence of coalitional and individual strategy-proofness properties, Soc. Choice Welfare 33 (2009) 287-309.

[13] H. Moulin, On strategy-proofness and single peakedness, Public Choice 35 (1980) 437-455.

[14] H. Moulin, Generalized Condorcet-winners for single peaked and single plateau preferences, Soc. Choice Welfare 1 (1984) 127-147. 
[15] H. Moulin, Incremental cost-sharing: Characterization by coalition strategy-proofness, Soc. Choice Welfare 16 (1999) 279-320.

[16] S. Pápai, Strategyproof assignment by hierarchical exchange, Econometrica 68 (2000) 1403-1433.

[17] P.K. Pattanaik, Strategy and Group Choice, North-Holland Publishing Company, 1978.

[18] B. Peleg, Almost all equilibria in dominant strategies are coalition-proof, Econ. Letters 60 (1998) 157-162.

[19] B. Peleg, P. Sudholter, Single-peakedness and coalition-proofness, Rev. Econ. Design 4 (1999) 381-387.

[20] A. Saporiti, Strategy-proofness and single-crossing, Theoretical Econ. 4 (2009) 127-163.

[21] M. Satterthwaite, Strategy-proofness and arrow's conditions: Existence and correspondence theorems for voting procedures and social welfare functions, J. Econ. Theory 10 (1975) 187-217.

[22] J. Schummer, R. Vohra, Strategy-proof location on a network, J. Econ. Theory 104 (2002) 405-428.

[23] A.K. Sen, P.K. Pattanaik, Necessary and sufficient conditions for rational choice under majority decision, J. Econ. Theory 1 (1969) 178-202.

[24] S. Serizawa, Pairwise strategy-proofness and self-enforcing manipulation, Soc. Choice Welfare 26 (2006) 305-331. 